\title{
Domain relaxation in Langmuir films
}

\author{
By JAMES C. ALEXANDER ${ }^{1}$, ANDREW J. BERNOFF \\ ELIZABETH K. MANN ${ }^{3}$, J. ADIN MANN Jr4, \\ JACOB R. WINTERSMITH ${ }^{5}$ AND LU ZOU ${ }^{3}$ \\ ${ }^{1}$ Department of Mathematics, Case Western Reserve University, Cleveland, OH 44106, USA \\ ${ }^{2}$ Department of Mathematics, Harvey Mudd College, Claremont, CA 91711, USA \\ ${ }^{3}$ Department of Physics, Kent State University, Kent, OH 44242, USA \\ ${ }^{4}$ Department of Chemical Engineering, Case Western Reserve University, Cleveland, OH 44106, USA \\ ${ }^{5}$ Department of Physics, Harvey Mudd College, Claremont, CA 91711, USA
}

(Received 1 November 2005 and in revised form 25 June 2006)

We report on theoretical studies of molecularly thin Langmuir films on the surface of a quiescent subfluid and qualitatively compare the results to both new and previous experiments. The film covers the entire fluid surface, but domains of different phases are observed. In the absence of external forcing, the compact domains tend to relax to circles, driven by a line tension at the phase boundaries. When stretched (by a transient applied stagnation-point flow or by stirring), a compact domain elongates, creating a bola consisting of two roughly circular reservoirs connected by a thin tether. This shape will then relax slowly to the minimum-energy configuration of a circular domain. The tether is never observed to rupture, even when it is more than a hundred times as long as it is wide. We model these experiments by taking previous descriptions of the full hydrodynamics, identifying the dominant effects via dimensional analysis, and reducing the system to a more tractable form. The result is a free boundary problem for an inviscid Langmuir film whose motion is driven by the line tension of the domain and damped by the viscosity of the subfluid. Using this model we derive relaxation rates for perturbations of a uniform strip and a circular patch. We also derive a boundary integral formulation which allows an efficient numerical solution of the problem. Numerically this model replicates the formation of a bola and the subsequent relaxation observed in the experiments. Finally, we suggest physical properties of the system (such as line tension) that can be deduced by comparison of the theory and numerical simulations to the experiment. Two movies are available with the online version of the paper.

\section{Introduction}

In this paper we develop a manageable model of the experimentally observed relaxation dynamics of a molecularly thin film with two fluid phases at an air/water interface. Our model refines previous work and is motivated by experimental observations. It is both analytically tractable and allows an efficient, accurate and stable numerical solution via a boundary integral technique. The model explains some observed experimental phenomena and in particular offers a more general method for measuring the line tension of the film. We neglect long-range electrostatic effects, which could be added in a straightforward manner at a later time, as well as viscosity and compressibility within the film. These approximations are reasonable for a wide but not universal range of experimental conditions, as discussed below. 

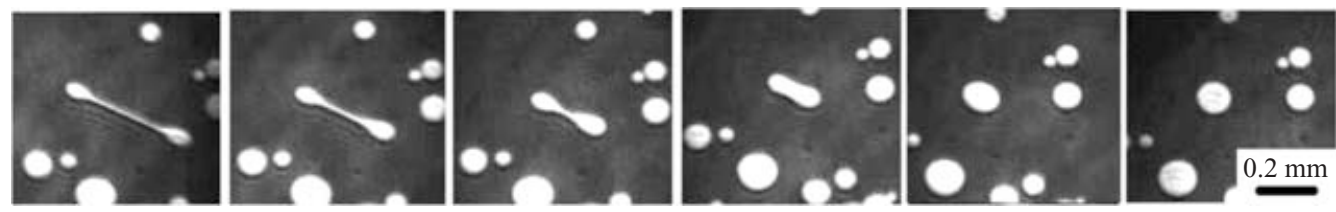

Figure 1. A series of Brewster Angle Microscopy photos showing a bola relaxing to a circular Langmuir domain. The brighter domains consist of about five layers of $8 \mathrm{CB}$, while the dark background consists of three layers of 8CB (de Mul \& Mann Jr 1998; Zou et al. 2006). A shear field was established which distorted the domain to a bola with a thin tether. The shear field was then shut off and the domain allowed to relax as shown in the series of images. The time interval is $0.5 \mathrm{~s}$. A short $(8 \mathrm{~s})$ movie of a Langmuir film being perturbed by stirring the subfluid and the resulting relaxation is available with the online version of the paper. The scale bar in the right frame spans $0.2 \mathrm{~mm}$.

\subsection{Langmuir films and line tension}

A Langmuir film is a molecularly thin layer bound at a fluid/gas interface so that the layer molecules do not escape into either fluid. Typically the fluid is water (or an aqueous solution) and the gas air. A balance of molecular interaction forces between the layer molecules, with each other and with the subfluid, leads to this molecularly thin layer. Depending on the surface density, the film may form quasitwo-dimensional analogues of a gas, liquid, liquid-crystal, solid, or other phases (Gaines Jr 1966). Multilayers of different thickness generate yet further possible phases. A thermodynamic equation of state for the Langmuir layer relates the surface pressure $\Pi$ and the surface density $\rho$. As in the three-dimensional case, the film phases can separate at intermediate average densities, into surface liquids with two different thicknesses. These phases form a distribution of separate domains at the surface. The phase coexistence region has drawn considerable experimental attention due to the wide variety of morphologies (Adamson \& Gast 1998) and dynamical behaviour that are potentially observable over a wide range of domain sizes from the nano- to the micro-scale. There is also a growing recognition of the functional importance of domains in biological cell membranes (Simons \& Ikonen 1997; Edidin 2003; Mayor \& Rao 2004; Parton \& Hancock 2004) for which Langmuir films can be a controlled model. As a film is compressed, domains of different thicknesses or composition acting as coexisting two-dimensional phases may appear.

This paper focuses on the case where there are two such co-existing liquid phase domains, which is particularly applicable to multilayer systems or to mixed monolayers, such as those found in biological membrane analogues. Note that bilayers and multilayers are explicitly included. The only assumption is that the set of layers is thin enough that the whole set can be considered to move together, and in particular that there is no slippage between layers. Such slippage is unlikely in an ordinary fluid layer. In three dimensions, slippage is perhaps seen in entangled polymers or in complex fluids.

Figure 1 shows a time-lapse set of Brewster Angle Microscopy (BAM) images that demonstrate the large aspect ratio of the typical bola that results from shearing a cyano-biphenyl liquid-crystal (8CB) Langmuir layer and its subsequent relaxation to a circular domain. Amazingly, these bola may be sheared to be several orders of magnitude longer than they are wide yet do not rupture. It is the physics behind this observation that we wish to model, explain and quantify

Figure 2 shows a cartoon of the cyano-biphenyl liquid crystal (8CB) studied by de Mul \& Mann Jr (1998) that provides a guide in developing our theory of 

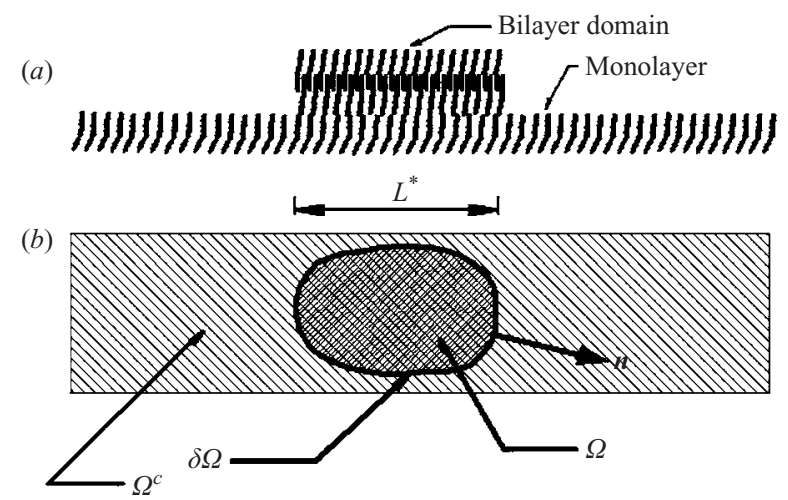

FiguRE 2. (a) An edge-on view of one set of two-dimensional fluid phases: an 8CB monolayer in coexistence with an $8 \mathrm{CB}$ trilayer. $8 \mathrm{CB}$ is a cyano-biphenyl molecule that forms a smectic (layered) liquid crystal at room temperature in bulk. (b) A top view of such a layer, defining the domain $\Omega$, the boundary $\partial \Omega$, the outer monolayer $\Omega^{c}$ and the normal $\hat{\boldsymbol{n}}$. See de Mul \& Mann Jr (1998).

domain behaviour. We assume that one phase is a localized domain, $\Omega$, and that its complement, $\Omega^{c}$, is a second phase which extends to infinity. The domain boundary $\partial \Omega$ will be parameterized by arclength $s$ with a right-handed orientation and with an outward pointing normal $\hat{\boldsymbol{n}}$.

Both phases behave as two-dimensional fluids. Each fluid can be characterized by a set of visco-elastic parameters, in direct analogy with the three-dimensional case (Goodrich 1981; Gaines Jr 1966; Mann Jr 1985; Mann Jr, Crouser \& Meyer 2001). The net attraction between film molecules, in the neighbourhood of the interface, leads to a line tension, or energy per unit length, $\lambda$, associated with the boundary between domains. Lateral intermolecular forces include short-range van der Waals forces, but also long-range dipolar repulsion due to the alignment of the effective molecular dipole moments by the interface.

Above a critical size $R_{c}$, given as

$$
R_{c}=\frac{\delta}{8} \exp \left[\frac{2 \pi \lambda}{\varepsilon_{0}(\Delta V)^{2}}+\frac{10}{3}\right]
$$

where $\delta$ is a characteristic molecular length, $\lambda$ is the line tension, $\varepsilon_{0}$ is the vacuum dielectric constant, and $\Delta V$ is the contrast in surface potential between the two phases, the long-range electrostatic forces will distort domains from the circular shape (DeKoker \& McConnell 1993; Mann, Hénon \& Langevin 1992). At dimensions much smaller than $R_{c}$, the major effect of the long-range forces is to renormalize the line tension as

$$
\lambda_{\mathrm{eff}}=\lambda-\mu^{2}\left(\ln \frac{L_{*}}{L_{m}}+\mathscr{I}_{s}\right),
$$

where $\mu$ is the effective dipole moment density difference, given in terms of the measured surface potential contrast as $\mu^{2}=\varepsilon_{0} / 2 \pi(\Delta V)^{2}, L_{*}$ is a typical domain length scale, $L_{m}$ is some molecular scale (often taken to be the thickness of the layer or the average distance between molecules in the layer), and $\mathscr{I}_{s}$ is a term depending only on shape (generally negligibly small until the $\lambda_{\text {eff }}$ approaches zero). Since the main correction to the line tension is logarithmic the line tension can often be taken as a constant over a large range of length scales. The long-range dipolar repulsion may 
still lead to repulsion between domains, and plausibly may help stabilize metastable states such as Langmuir foams (Mann et al. 1992). Between them, the line tension and the electrostatic effects determine both the equilibrium and dynamic behaviour of the domains. In particular, both the characteristic size and shape of the domains will depend on these factors. The electrostatic effects can be determined experimentally by measuring the surface potential, or the drop in voltage across the interface, due to the alignment of molecular dipoles (Mann 1992; Mann et al. 1992). Determining the line tension has been more difficult, and relatively few examples exist in the literature.

\subsection{Previous results, experiments, and background}

The existence of a line tension means that small isolated domains are round in equilibrium, and also that deformed domains relax to this shape, as can be seen in figure 1. In order to deform the domain, a shear is applied to the underlying liquid, and then removed. Typically highly deformed states look like bola, with a tether connecting two nearly circular ends which approach each other with a speed which depends on the line tension and the viscosity of both the Langmuir film and the underlying fluid. As the two ends approach each other closely, the tether thickens and eventually disappears as the domain relaxes finally towards a circle.

\subsubsection{Line tension}

Benvegnu \& McConnell (1992) estimated the line tension in a mixed monolayer from the speed of approach of the two bola ends, using a simple hydrodynamic approximation assuming a negligible surface viscosity and circular bola with no flow at the interior, using the results of Hughes, Pailthorpe \& White (1981) for a solid cylinder moving through a membrane. Once the domain shape had become convex, the relaxation was found to be exponential (cf. Mann et al. 1992, 1995), as is expected in the small deformation limit. The line tension for different systems was deduced from this relaxation by Mann et al. (1992), Mann et al. (1995) and Lauger et al. (1996) using a hydrodynamic approximation developed by Stone \& McConnell (1995). Results were consistent with line tension deduced from the bola velocities. Similarly, line tension estimates were obtained from the coalescence, and subsequent relaxation, of two domains (Mann et al. 1992; Steffen, Wurlitzer \& Fischer 2001). In other experiments, the domains were instead deformed directly using silica beads at the domain edges as handles for optical tweezers (Wurlitzer, Steffen \& Fischer 2000a; Wurlitzer et al. 2000b).

Mathematical models of a Langmuir film as a viscous two-dimensional fluid on a viscous three-dimensional subfluid were developed by Stone \& McConnell (1995) and Lubensky \& Goldstein (1996). These models assumed that the surface layer is infinite and of constant viscosity, with the domain advected by the surface flow and a force applied via the line tension. Specifically, Stone \& McConnell (1995) included strong electrostatic repulsion, and found growth rates for small deformations, for any ratio between the surface and volume viscosities. From their results, relaxation rates for the case where line tension dominates electrostatic repulsion can be deduced. Lubensky \& Goldstein (1996) developed a Green's function approach, again assuming that the surface viscosity is a constant across the entire Langmuir layer. While both these calculations allowed for a finite-depth subfluid, under experimental conditions the subfluid is usually much deeper than the size of the domains, and we will assume that it is effectively infinitely deep.

A major goal of this work is to develop a theoretical framework to determine the experimental line tension between two fluid phases. Direct measurements of 
the relaxation time for perturbations from a circular domain as a function of volume viscosity (Mann et al. 1995) for a polymer monolayer (polydimethylsiloxane, PDMS) on water demonstrated that relaxation can be dominated by viscosity in the subfluid and allowed estimation of the line tension. However, the regime of very small deformations is experimentally difficult to access. Ideally, the whole range of relaxation behaviour could be used in the line tension determination.

\subsubsection{Tethers}

A further motivation for our research concerns the tether, that is, the long thin line of fluid between the two rounded ends of a bolus. Tethers are inherently unstable in pure three-dimensional fluids because of the Rayleigh instability (cf. Drazin \& Reid 2004); varicose (peristaltic) mode fluctuations decrease the surface area, and thus the energy of the system. Such tethers may nevertheless play important roles in fluid membranes, such as in biological cells, where they may be stabilized by the elastic properties of the membranes as was shown by Powers, Huber \& Goldstein (1990).

For a two-dimensional tether, the stability question is much more subtle; one natural analogy is with an idealized thin fluid soap film, which is linearly stable to capillary forces, as small perturbations now increase the surface area (cf. Drazin \& Reid 2004). However in a real soap film a variety of surface effects must be included to fully capture the dynamics. For example, the viscoelastic response of the surfactant layers bounding the film can slow draining of the film under gravity. Static considerations, such as the competing effects of electrostatic repulsion and van der Waals attraction between the two surfaces of the film, can also affect whether the film ruptures. The analysis of thin lines of two-dimensional fluid in monolayers (Lucassen, Akamatsu \& Rondelez 1991) has suggested the need for a 'surfactant' that adsorbs on the boundaries of the thin line or tether. Brochard-Wyart (1990) on the other hand suggests that van der Waals attraction is sufficiently small that the characteristic 'soap film' peristaltic instability would develop only over very long times.

By direct experimental comparison, Mann \& Primak (1999) demonstrated that the two-dimensional soap film is much less stable in the absence of direct electrostatic dipolar repulsion, owing to the alignment of molecular dipoles. While this work suggests that one way to stabilize a tether is to incorporate dipolar repulsive forces between the interfaces, our model suggests that a tether can be stable in the absence of these forces.

Whereas the soap film provides one analogue for the stability of a tether, a sharply contrasting one is the nearly two-dimensional flow of the Hele-Shaw problem (Almgren 1996; Glasner 2003), a mathematical model for the evolution of a viscous fluid droplet sandwiched in a narrow gap between two plates. It is known that this flow also evolves to minimize the perimeter of the bubble, so modestly deformed domains relax to being circular. However, it is known numerically and asymptotically that an initially long narrow bubble will first relax into a bola configuration with a narrow neck forming between the circular reservoir and the tether (see Glasner 2003). This neck will eventually pinch off (Almgren 1996; Almgren, Bertozzi \& Brenner 1996; Constantin et al. 1993; Dupont et al. 1993; Glasner 2003; Goldstein, Pesci \& Shelley 1993) in a finite time, leading naturally to the question of why the dynamics of Langmuir layers are different. This question is explored herein.

\subsection{A free boundary formulation}

Another goal of our analysis is to reduce the equations of motion for the Langmuir layer and the subfluid to a simpler description for the evolution of the boundary 
of the domain. Our dimensional analysis below will indicated that the pressure in the Langmuir layer is in a hydrostatic balance while the subfluid is Stokesian. Consequently, the whole system is quasi-steady; that is the velocity of the subfluid and the Langmuir layer can be determined solely in terms of the instantaneous location of the domain boundary. Moreover, this response is linear. Consequently, the evolution of the boundary can be described by a boundary integral formulation (Pozrikidis 1992); that is the boundary's velocity at each point can be determined as a convolution integral over the boundary. Once again, this is analogous to the Hele-Shaw problem, which allows a similar formulation (e.g. Tryggvason \& Aref 1983). Previously a boundary integral formulation for the Langmuir layer (including electrostatic forces) had been proposed by Lubensky \& Goldstein (1996) and an analogous version was implemented by Heinig, Helseth \& Fischer (2004), who were able to qualitatively reproduce many experimental results, although their scheme exhibits modest area loss. We use a different formulation of the free boundary problem which lends itself to a more accurate numerical implementation. Both the Hele-Shaw problem and our description of the Langmuir layer are driven by curvature, which leads to numerical stiffness; we address this problem using a semi-implicit pseudospectral method developed by Hou, Lowengrub \& Shelley (1994) for Hele-Shaw flow. This formulation allows simulation of the stretching, formation and subsequent relaxation of a bola in a Langmuir layer. We are able to simulate relaxation of tethers with aspect ratios of over 100-to-1 with good control of error.

\subsection{Outline of the paper}

Experimentally, Langmuir layers often separate into different phases on the surface of the fluid. In $\S 2$, we develop a model for a Langmuir layer consisting of two fluid phases. We call such regions Langmuir layer domains and concentrate on one such domain, $\Omega$, and its complement, $\Omega^{c}$, which is assumed to be a different phase. We model these domains as incompressible inviscid two-dimensional Newtonian fluids. We also assume that the line-tension $\lambda$ along the boundary of $\Omega$ is constant. Dimensional analysis of our experiments suggests that, at leading order, the subfluid is Stokesian and the Langmuir layer is inviscid. Hence we call the model the inviscid Langmuir layer Stokesian subfluid (ILLSS) model. Basically the line tension drives the motion, which is damped by the viscosity of the subfluid, dissipating the energy. Our dimensional analysis also suggests that at leading order the surface of the subfluid, on which the Langmuir layer lies, is flat, and that the film has negligible thickness.

In the special case when the Langmuir domains have the same surface viscosity, a solution can be found in which the subfluid has constant pressure and all motion is in horizontal planes (an ansatz made in the works of Stone \& McConnell 1995 and Lubensky \& Goldstein 1996). In this case, we introduce a streamfunction formulation which greatly simplifies the analysis.

In $\S 3$ we consider the energy balance in the fluid and show that the modelled system dissipates energy by decreasing the length of the boundary of $\Omega$. This suggests that, for any initial configuration, $\Omega$ relaxes to a circular domain or possibly a collection of disjoint circular domains.

In $\$ 4$ we consider some details of the final stage of the relaxation to a circular domain via a linear stability analysis. Our analysis reproduces the known results for relaxation to circular domains in a fairly streamlined fashion.

As a simple model of a long narrow tether, in $\S 5$ we consider linear stability of an infinite strip. Not surprisingly, we discover that such a strip is linearly stable to both varicose and sinuous instabilities. 


\begin{tabular}{|c|c|c|c|}
\hline Parameter & Symbol & Range of values & Comments \\
\hline Length & $L_{*}$ & $5 \mu \mathrm{m}<L_{*}<5 \mathrm{~mm}$ & Optical microscopy limit \\
\hline Surface tension & $\sigma$ & $10-72 \mathrm{mN} \mathrm{m}^{-1}$ & Experimental measurements \\
\hline Line tension & $\lambda$ & $\leqslant 1-20 \mathrm{pN}$ & Experimental measurements \\
\hline Volume viscosity & $\eta^{\prime}$ & $>10^{-3} \mathrm{~kg} \mathrm{~m}^{-1} \mathrm{~s}^{-1}$ & Water plus additives \\
\hline Surface viscosity & $\eta$ & $\left\{\begin{array}{l}\left(<10^{-8}\right)-10^{-1} \mathrm{~kg} \mathrm{~s}^{-1} \\
<10^{-8} \mathrm{~kg} \mathrm{~s}^{-1} \\
10^{-7} \mathrm{~kg} \mathrm{~s}^{-1} \\
10^{-6} \mathrm{~kg} \mathrm{~s}^{-1} \\
10^{-3} \mathrm{~kg} \mathrm{~s}^{-1}\end{array}\right.$ & $\begin{array}{l}\text { Experimental results : } \\
\text { - polymer(PDMS) } \\
\text { - liquid expanded }{ }^{\dagger} \\
\text { - liquid condensed } \\
\text { - } \text { solid }^{\dagger}\end{array}$ \\
\hline Domain thickness & $\xi$ & $0.5-10 \mathrm{~nm}$ & Mono- to multi-layers \\
\hline Volume density & $\rho^{\prime}$ & $10^{3} \mathrm{~kg} / \mathrm{m}^{3}$ & Water \\
\hline Surface density & $\rho$ & $(1-20) \times 10^{-6} \mathrm{~kg} \mathrm{~m}^{-2}$ & Mono- to multi-layers \\
\hline Vertical deformation radius & $\lambda / \sigma$ & $\left\{\begin{array}{l}\leqslant \xi<10 \mathrm{~nm} \\
<1 \mathrm{~nm}\end{array}\right.$ & $\begin{array}{l}\text { Theory } \\
\text { Experiment }\end{array}$ \\
\hline $\begin{array}{l}\text { Reynolds number } \\
\text { Density ratio } \\
\text { Viscosity ratio }\end{array}$ & $\begin{array}{l}\mathrm{Re}=\rho^{\prime} \lambda / \eta^{\prime 2} \\
\delta_{\rho}=\rho / \rho^{\prime} L_{*} \\
\delta_{\eta}=\eta / \eta^{\prime} L_{*}\end{array}$ & $\begin{array}{l}\lesssim 10^{-2} \\
\sim 10^{-3} \\
10^{-2}-10^{3}\end{array}$ & \\
\hline
\end{tabular}

TABLE 1. Physical and non-dimensional parameters for Langmuir layers from theory and experiment. For references see: ${ }^{\dagger}$ Joly (1972), ${ }^{\ddagger}$ Mann et al. (1995).

In $\S 6$ we derive a boundary integral formulation of the ILLSS model that allows an efficient numerical implementation of the nonlinear evolution of a bola. We show numerically that the stretching of a Langmuir layer domain by a transient stagnation-point flow leads to the formation of a bola and subsequent relaxation back to a circular domain, qualitatively reproducing the experimental observations. Our numerics suggest that existing heuristic theories that deduce line tensions from tether relaxation rates are accurate to roughly $10 \%$.

Finally, in $\S 7$ we discuss how this model can be used to deduce the line tension on a Langmuir layer domain by comparing the experiments to the numerical and analytical results.

\section{Formulation}

\subsection{Dominant balance in the Langmuir layer and subfluid}

As the subfluid in the experiment is usually water or some aqueous solution, we assume its evolution will be governed by the Navier-Stokes equation for an incompressible fluid characterized by its density, $\rho^{\prime}$, and viscosity, $\eta^{\prime}$. Moreover, following previous authors, we will also assume that the Langmuir layer can be modelled as a twodimensional Newtonian fluid with associated surface density, $\rho$, and surface viscosity, $\eta$. The two are coupled at the surface by the applied stress balance; the subfluid's surface tension acts normal to the surface, the line tension acts tangent to the surface (and normal to the domain's boundary), and viscous normal and tangential stresses can be found in both directions. Table 1 provides a list of physical parameters for our experiment and their range of values as deduced from both theory and experimental observation. We justify our approximation with dimensional analysis below. 


\subsubsection{Stokesian subfluid}

We now propose the ansatz that the relaxation of a polymer domain from, say, a bola shape to a circular patch is driven by the line tension, $\lambda$, of the patch and that the energy is dissipated by the viscosity, $\eta^{\prime}$, of the subfluid. Since the domain $\Omega$ is incompressible, say with fixed area $A_{*}$, we can choose a linear characteristic domain size, $L_{*} \sim \sqrt{A_{*}}$, and non-dimensionalize the problem based on a characteristic length, time and mass,

$$
L_{*}, \quad T_{*}=\frac{\eta^{\prime}\left(L_{*}\right)^{2}}{\lambda}, \quad M_{*}=\eta^{\prime} L_{*} T_{*}=\frac{\left(\eta^{\prime}\right)^{2}\left(L_{*}\right)^{3}}{\lambda},
$$

respectively.

In the bulk of the subfluid, we assume the non-dimensional fluid velocity, $\boldsymbol{u}=$ $u \hat{\boldsymbol{\imath}}+v \hat{\boldsymbol{\jmath}}+w \hat{\boldsymbol{k}}$, is incompressible

$$
\nabla \cdot \boldsymbol{u}=0
$$

and satisfies the non-dimensional Navier-Stokes equations

$$
R e\left(\boldsymbol{u}_{t}+\boldsymbol{u} \cdot \nabla \boldsymbol{u}\right)=-\nabla P+\nabla^{2} \boldsymbol{u},
$$

where $P$ is the non-dimensional pressure and the Reynolds number

$$
R e=\frac{\rho^{\prime} \lambda}{\left(\eta^{\prime}\right)^{2}}
$$

is assumed to be small; for an aqueous substrate and with a Langmuir-layer line tension $\lambda<10^{-11} \mathrm{~N}$ we find $R e \lesssim 2 \times 10^{-2}$.

Consequently, the subfluid velocity satisfies the Stokes equations

$$
\nabla P=\nabla^{2} \boldsymbol{u}
$$

at leading order.

\subsubsection{Normal stress balance on the surface}

We next consider the normal stress balance at the surface. If we allow the surface to deform, we need to balance surface tension, pressure and viscous stresses from the subfluid, viscous stresses from the Langmuir layer and geometrical contributions from the deforming interface (Aris 1990; Stone \& McConnell 1995). However, we argue that at leading order the surface remains flat. To estimate the deformation, we assume that the viscous stresses in the film are smaller than or comparable with the viscous stresses in the subfluid. Also, the constant atmospheric pressure $P_{0}$ can be eliminated by subtracting a constant from the pressure in the fluid. The normal component of the fluid's stress tensor (which has contributions from the pressure and viscosity) will balance the surface tension. With our non-dimensionalization, we find that at the surface,

normal viscous stress $\sim$ surface tension,

$$
\frac{\eta^{\prime}}{T_{*}} \sim \sigma \mathscr{H}
$$

where $\sigma$ is the surface energy (which may depend upon whether it is inside or outside the domain) and $\mathscr{H}$ is the mean curvature of the surface. We can solve for the magnitude of the surface curvature,

$$
\mathscr{H} \sim \frac{\lambda}{\sigma\left(L_{*}\right)^{2}} .
$$


For

$$
L_{*} \gg \lambda / \sigma
$$

we discover that the radius of curvature of the surface is much larger than the typical domain size; typically $\lambda / \sigma \approx 10 \mathrm{~nm}$ so we are looking at domains $10-1000$ times larger than this length scale. Consequently, we consider the case of a flat surface with the subfluid occupying the region $z<0$ and the Langmuir layer domain $\Omega$ contained in the $x, y$ coordinate plane, $z=0$, as illustrated in figure 2 .

\subsubsection{Tangential stress balance in the Langmuir layer domain}

For a flat surface, the Langmuir layer evolution equations simplify drastically. Balancing the tangential stresses on the surface yields a force applied by the subfluid on the domain $\Omega$. The non-dimensional stress tensor for our viscous, incompressible Newtonian fluid is

$$
\boldsymbol{T}=-P \boldsymbol{I}+\nabla \boldsymbol{u}+(\nabla \boldsymbol{u})^{T} .
$$

The tangential stress at the surface acts as a two-dimensional body force, $\boldsymbol{F}_{s}$, (specifically, a force per unit area) acting on the Langmuir layer,

$$
\boldsymbol{F}_{s}=-\hat{\boldsymbol{k}} \cdot \boldsymbol{T} \cdot(\boldsymbol{I}-\hat{\boldsymbol{k}} \hat{\boldsymbol{k}})=-\left[u_{z} \hat{\boldsymbol{\imath}}+v_{z} \hat{\boldsymbol{J}}\right] .
$$

As noted above, the normal component of stress at the surface is balanced by surface tension and produces a negligible deformation of the surface. We assume that the Langmuir layer domain acts like a two-dimensional Newtonian fluid, with this applied body force.

\subsection{The inviscid Langmuir layer}

Using the same non-dimensionalization for the Langmuir-layer surface velocity, $\boldsymbol{U}=$ $U \hat{\boldsymbol{\imath}}+V \hat{\boldsymbol{J}}$, and pressure, $\Pi$, we see that the velocity field is that of an incompressible fluid,

$$
\nabla_{\perp} \cdot \boldsymbol{U}=0
$$

where $\nabla_{\perp} \cdot$ is the surface divergence. The surface momentum balance yields the twodimensional Navier-Stokes equation,

$$
\delta_{\rho} \operatorname{Re}\left(\boldsymbol{U}_{t}+\left(\boldsymbol{U} \cdot \nabla_{\perp}\right) \boldsymbol{U}\right)=\boldsymbol{F}_{s}+\boldsymbol{F}_{\ell}-\nabla_{\perp} \Pi+\delta_{\eta} \nabla_{\perp}^{2} \boldsymbol{U},
$$

where $\nabla_{\perp}$ is the surface gradient and $\boldsymbol{F}_{\ell}$ is the force associated with the line tension. Following Stone \& McConnell (1995) and Lubensky \& Goldstein (1996), we model $\boldsymbol{F}_{\ell}$ as a line force on $\partial \Omega$ proportional to the curvature; a specific form is given below.

Assuming a water substrate, we find that

$$
\delta_{\rho}=\frac{\rho}{\rho^{\prime} L_{*}} \lesssim 10^{-3}
$$

represents the mass ratio of a portion of the Langmuir layer to the fluid in motion beneath it; consequently, the inertial forces in the Langmuir layer domain are smaller than those in the subfluid and are neglected. The ratio

$$
\delta_{\eta}=\frac{\eta}{\eta^{\prime} L_{*}}
$$

represents the ratio of viscous dissipation in the surface to viscous dissipation in the subfluid; when

$$
L_{*} \gg \frac{\eta}{\eta^{\prime}}
$$


we can also neglect the viscous dissipation in the film. The viscosity ratio depends strongly on the Langmuir layer and its phases, as can be seen in table 1. The surface viscosity has been found experimentally to be negligible for several fluid phases (Mann et al. 1995; Lauger et al. 1996), most explicitly for a polymer (PDMS) where the ratio was found to be $\ll 10 \mu \mathrm{m}$. The parameter can also be adjusted to be as small as desired by increasing the viscosity of the substrate, which is possible with water/glycerol mixtures (Mann et al. 1995).

Henceforth we assume that inertial effects and viscous dissipation in the Langmuir layer are subdominant, so the dominant balance is between the surface pressure, the applied stress from the subfluid, and the line tension. Balancing the forces with the pressure, we see that

$$
\nabla_{\perp} \Pi=\boldsymbol{F}_{s}+\boldsymbol{F}_{\ell},
$$

which means the Langmuir layer is in hydrostatic equilibrium.

To describe the line tension we develop an intrinsic coordinate system on the surface near the boundary of the domain (see figure $2 b$ ). Let $\boldsymbol{\Gamma}(s, t)$ be a position vector for the boundary of the domain, $\partial \Omega$, parameterized by the arclength $s$. We choose a right-handed orientation; that is, moving along $\partial \Omega$ counterclockwise with $\Omega$ to the left corresponds to increasing $s$. Let $\hat{t}$ be the corresponding tangent vector. Differentiating with respect to arclength yields $\mathrm{d} \hat{\boldsymbol{t}} / \mathrm{d} s=\kappa \hat{\boldsymbol{n}}$, where $\hat{\boldsymbol{n}}$ is the outwardpointing normal vector and $\kappa$ is the curvature of $\partial \Omega$ (negative for convex $\Omega$ ). We define the signed distance from $\partial \Omega$ along $\hat{\boldsymbol{n}}$ as $d$ with $d<0$ in the interior of $\Omega$ and $d>0$ in the exterior $\left(\Omega^{c}\right)$. For sufficiently small $|d|$, the contours $\{(x, y): d(x, y)=d\}$ are curves equidistant from the original $\partial \Omega$, and coordinates for a neighbourhood of $\partial \Omega$ can be given by the pair $(s, d)$. We can now rewrite the position vector to a point on the surface in terms of $(s, d)$ :

$$
\boldsymbol{R}(s, d)=x(s, d) \hat{\boldsymbol{\imath}}+y(s, d) \hat{\boldsymbol{J}}=\boldsymbol{\Gamma}(s, t)+d \hat{\boldsymbol{n}} .
$$

Now, the line tension force can be written as

$$
\boldsymbol{F}_{\ell}=\kappa \hat{\boldsymbol{n}} \delta(d),
$$

where $\delta(d)$ is a measure supported on $\partial \Omega$ that is a delta function for any curve crossing $\partial \Omega$ transversally. Thus, if we integrate across the boundary, we find that the pressure jump

$$
[\Pi]_{d=-\epsilon}^{d=\epsilon}=\kappa
$$

is proportional to the curvature, as expected.

\subsection{The kinematic condition}

To complete the formulation we use the kinematic condition to tie the motion of the surface to that of the subfluid. As the surface is flat, the vertical velocity at the surface vanishes,

$$
w(x, y, 0)=0 .
$$

In addition, we assume continuity of the tangential velocity at the surface,

$$
\boldsymbol{U}=U(x, y) \hat{\boldsymbol{\imath}}+V(x, y) \hat{\boldsymbol{J}}=u(x, y, 0) \hat{\boldsymbol{\imath}}+v(x, y, 0) \hat{\boldsymbol{J}} .
$$

Finally, we also note that the boundary of the domain is advected with the surface velocity,

$$
\frac{\mathrm{D} \boldsymbol{\Gamma}}{\mathrm{D} t}=\left.\boldsymbol{U}\right|_{\partial \Omega},
$$

where $\mathrm{D} \boldsymbol{\Gamma} / \mathrm{D} t$ is the material derivative of a point on the boundary. 


\subsection{The inviscid Langmuir-layer Stokesian subfluid approximation (ILLSS)}

Under the assumptions that the Langmuir layer is inviscid and the subfluid is Stokesian, a complete set of governing equations can be developed. The subfluid velocity satisfies the Stokes equations

$$
\begin{aligned}
& \nabla \cdot \boldsymbol{u}=0, \quad z<0, \\
& \nabla^{2} \boldsymbol{u}=\nabla P, \quad z<0 .
\end{aligned}
$$

In experimental conditions, a Langmuir layer domain, $\Omega$, occupies a small portion of the surface area of the subfluid. Thus we assume that the subfluid extends infinitely far in the horizontal $r=\sqrt{x^{2}+y^{2}}$ and vertical $z<0$ directions. If we assume the domain is finite in extent, the force it applies to the surface is localized and the response of the subfluid will decay algebraically in $r$ and exponentially in $z$ (see Stone \& McConnell 1995); consequently, we can assume that the velocity $\boldsymbol{u}$ and its gradients decay uniformly to 0 at $\infty$ :

$$
|\boldsymbol{u}|, \quad|\nabla \boldsymbol{u}|, \quad\left|\nabla^{2} \boldsymbol{u}\right| \rightarrow 0 \quad \text { as } \quad r^{2}+z^{2} \rightarrow \infty .
$$

A second possibility is that the region of interest is embedded in an external flow, such as a transient straining field. We simplify the problem by assuming that the external flow, $\boldsymbol{U}_{\text {ext }}$, is irrotational, and uniform in the vertical direction,

$$
\boldsymbol{U}_{\text {ext }}=U_{\text {ext }}(x, y) \hat{\boldsymbol{\imath}}+V_{\text {ext }}(x, y) \hat{\boldsymbol{\jmath}}, \quad\left(V_{\text {ext }}\right)_{x}-\left(U_{\text {ext }}\right)_{y}=0 .
$$

In this case, an appropriate boundary condition is that the velocity deviation from the imposed flow, $\boldsymbol{u}-\boldsymbol{U}_{\text {ext }}$, and its gradients vanish far from the domain boundary.

The tangential surface stress, $\boldsymbol{F}_{s}$, and line tension, $\boldsymbol{F}_{\ell}$, are in hydrostatic equilibrium with the surface pressure:

$$
\begin{aligned}
\nabla_{\perp} \Pi & =\boldsymbol{F}_{s}+\boldsymbol{F}_{\ell} \\
& =-u_{z}(x, y, 0) \hat{\boldsymbol{\imath}}-v_{z}(x, y, 0) \hat{\boldsymbol{\jmath}}+\kappa \hat{\boldsymbol{n}} \delta(d) .
\end{aligned}
$$

Also, the surface is incompressible:

$$
\nabla_{\perp} \cdot \boldsymbol{U}=u_{x}(x, y, 0)+v_{y}(x, y, 0)=0,
$$

which guarantees the area of the domain is conserved. Finally, the kinematic condition implies that the normal velocity vanishes at the surface:

$$
w(x, y, 0)=0
$$

and that the boundary of the domain is advected with the surface velocity:

$$
\frac{\mathrm{D} \boldsymbol{\Gamma}}{\mathrm{D} t}=\left.\boldsymbol{u}\right|_{\partial \Omega} .
$$

Equations (2.18)-(2.26) completely specify the system.

\subsection{Streamfunction formulation for the ILLSS approximation}

As noted by both Stone \& McConnell (1995) and Lubensky \& Goldstein (1996), a significant simplification can be obtained when motion of the fluid is confined to horizontal layers; typically this is possible when the Langmuir layer is incompressible and the viscosities in $\Omega$ and $\Omega^{c}$ are equal. In the present case, the viscosities are both zero and the ILLSS model has a solution of this form. 


\subsubsection{A horizontal flow solution}

We begin by introducing a streamfunction $\psi_{\text {ext }}(x, y)$ for the external flow

$$
\boldsymbol{U}_{\mathrm{ext}}=\hat{\boldsymbol{k}} \times \nabla_{\perp} \psi_{\mathrm{ext}}=-\left(\psi_{\mathrm{ext}}\right)_{y} \hat{\boldsymbol{\imath}}+\left(\psi_{\mathrm{ext}}\right)_{x} \hat{\boldsymbol{J}} .
$$

Note that as the external flow is irrotational and two-dimensional, the vertical vorticity vanishes:

$$
\hat{\boldsymbol{k}} \cdot \nabla \times \boldsymbol{U}_{\mathrm{ext}}=\nabla_{\perp}^{2} \psi_{\mathrm{ext}}=0,
$$

where $\nabla_{\perp}^{2}=\partial_{x x}^{2}+\partial_{y y}^{2}$ is the horizontal Laplacian.

Next, we look for a solution for the velocity deviation of the subfluid of the form

$$
\boldsymbol{u}-\boldsymbol{U}_{\mathrm{ext}}=\hat{\boldsymbol{k}} \times \nabla_{\perp} \psi=-\psi_{y} \hat{\boldsymbol{\imath}}+\psi_{x} \hat{\boldsymbol{\jmath}}, \quad P=P_{0},
$$

where $\psi=\psi(x, y, z)$ is a streamfunction for the motion in horizontal planes. In this case, we discover that the fluid automatically satisfies incompressibility. Taking the curl of (2.19) shows that the vertical vorticity $\hat{\boldsymbol{k}} \cdot \nabla \times \boldsymbol{u}=-\nabla_{\perp}^{2} \psi$ is harmonic in three dimensions and consequently the streamfunction $\psi$ satisfies the 'twice-harmonic' equation

$$
\nabla_{\perp}^{2}\left(\nabla^{2} \psi\right)=0
$$

in the subfluid.

From the decay of the velocity deviation in the far field, (2.20), we see that $\nabla^{2} \psi \rightarrow 0$ as $r \rightarrow \infty$. Also, we can deduce from the fact that $\nabla_{\perp}^{2}\left(\nabla^{2} \psi\right)=0$ (that is that $\nabla^{2} \psi$ is harmonic in each horizontal plane) and Liouville's Theorem from the analysis of a complex variable (which states that any harmonic function bounded at infinity must be constant) that

$$
\nabla^{2} \psi=f(z)
$$

for some function $f(z)$. A gauge transformation, $\psi \rightarrow \psi+g(z)$ with $g_{z z}=f$ allows us to find a solution for $\psi$ that is harmonic in three dimensions,

$$
\nabla^{2} \psi=0 .
$$

The decay of the velocity deviation (2.20) implies that $|\nabla \psi| \rightarrow 0$ as $r^{2}+z^{2} \rightarrow \infty$.

Incompressibility of the fluid (2.18) specifies $\nabla_{\perp} \cdot \boldsymbol{u}=0$ in every layer, and at the surface

$$
0=-\left(\nabla_{\perp} \cdot \boldsymbol{u}\right)_{z}=-\nabla_{\perp} \cdot \boldsymbol{u}_{z}=\nabla_{\perp} \cdot \boldsymbol{F}_{s}=\nabla_{\perp}^{2} \Pi-\nabla_{\perp} \cdot \boldsymbol{F}_{\ell} .
$$

Consequently, the surface pressure $\Pi$ is determined by

$$
\nabla_{\perp}^{2} \Pi=\nabla_{\perp} \cdot \boldsymbol{F}_{\ell}
$$

where we also specify that $|\Pi| \rightarrow 0$ far from $\Omega$.

\subsubsection{Surface-stress streamfunction}

We now introduce a streamfunction for the surface stress,

$$
S(x, y)=\psi_{z}(x, y, 0),
$$

so the tangential stress balance equation (2.22) becomes (see equation (2.9))

$$
\boldsymbol{F}_{s}=-u_{z} \hat{\boldsymbol{\imath}}-v_{z} \hat{\boldsymbol{J}}=-\psi_{y z} \hat{\boldsymbol{\imath}}+\psi_{x z} \hat{\boldsymbol{J}}=-\hat{\boldsymbol{k}} \times \nabla \psi_{z}=-\hat{\boldsymbol{k}} \times \nabla S,
$$

so that

$$
-\hat{\boldsymbol{k}} \times \nabla S=\nabla \Pi-\boldsymbol{F}_{\ell} .
$$


Note from (2.33) that $\Pi$ is harmonic in both $\Omega$ and $\Omega^{c}$, and from (2.35) that $S$ and $\Pi$ are harmonic conjugates in these regions, so $S$ must be harmonic also. If we take the vertical component of the curl of (2.35), we find that

$$
\begin{aligned}
\nabla^{2} S & =-\nabla_{\perp} \cdot\left(\hat{\boldsymbol{k}} \times \boldsymbol{F}_{\ell}\right) \\
& =-\nabla_{\perp} \cdot(\kappa \hat{\boldsymbol{t}} \delta(d)) \\
& =-\kappa_{s} \delta(d),
\end{aligned}
$$

where $\kappa_{s}$ is the derivative of the curvature with respect to arclength.

To summarize, in the subfluid, we seek a harmonic streamfunction that satisfies the surface stress balance

$$
\begin{aligned}
\nabla^{2} \psi & =0, \quad z<0, \\
\psi_{z} & =S,
\end{aligned}
$$

and $|\nabla \psi| \rightarrow 0$ as $z \rightarrow-\infty$. The surface stress satisfies

$$
\nabla_{\perp}^{2} S=-\kappa_{s} \delta(d)
$$

and vanishes in the far field. Finally, the boundary of the domain is advected with the velocity of the subfluid evaluated at the surface:

$$
\frac{D \boldsymbol{\Gamma}}{D t}=\boldsymbol{U}_{\text {ext }}+\hat{\boldsymbol{k}} \times\left.\nabla_{\perp} \psi\right|_{z=0} \quad \text { on } \quad \partial \Omega,
$$

Equations (2.39)-(2.42) specify the evolution of the domain completely. Moreover, computing the boundary velocity has been reduced to solving Laplace's equation twice, once on the two-dimensional surface and once in the three-dimensional subfluid in the half-space $z<0$.

\section{Energy and energy dissipation}

In this section we show that in the absence of an external flow $\left(\boldsymbol{U}_{\text {ext }}=0\right)$ the ILLSS model dissipates energy by reducing the arclength of the boundary of the domain. The length of the boundary $L$ is

$$
L(t)=\oint_{\partial \Omega} \mathrm{d} s
$$

Then,

$$
L_{t}=-\oint_{\partial \Omega} \kappa(\boldsymbol{U} \cdot \hat{\boldsymbol{n}}) \mathrm{d} s,
$$

where we have used the fact that the boundary of the domain is advected materially with the surface velocity $\boldsymbol{U}$ evaluated on the boundary of the domain $\partial \Omega$. Since the curvature $\kappa$ equals the jump in the surface pressure on the domain boundary, we can use the definition of the delta function to extend the integral to the entire surface. Substituting and using the divergence theorem yields

$$
\begin{aligned}
L_{t} & =-\oint_{\partial \Omega} \kappa(\boldsymbol{U} \cdot \hat{\boldsymbol{n}}) \mathrm{d} s \\
& =-\iint_{z=0} \boldsymbol{U} \cdot(\kappa \delta(d) \hat{\boldsymbol{n}}) \mathrm{d} x \mathrm{~d} y \\
& =-\iint_{z=0} \boldsymbol{U} \cdot\left(\nabla_{\perp} \Pi-\boldsymbol{F}_{s}\right) \mathrm{d} x \mathrm{~d} y .
\end{aligned}
$$


Note that if we integrate the first term over a circle of radius $R$, then

$$
\begin{aligned}
\iint_{r<R} \boldsymbol{U} \cdot \nabla_{\perp} \Pi \mathrm{d} x \mathrm{~d} y & =\iint_{r<R} \nabla_{\perp} \cdot(\Pi \boldsymbol{U}) \mathrm{d} x \mathrm{~d} y \\
& =\oint_{r=R} \Pi(\boldsymbol{U} \cdot \hat{\boldsymbol{n}}) \mathrm{d} s \\
& =O(1 / R),
\end{aligned}
$$

where the first step employs the incompressibility of the Langmuir layer, and we have used the fact that the pressure and the velocity are $O(1 / R)$ in the far field. Consequently, this term vanishes as $R \rightarrow \infty$.

Rewriting the remaining term in terms of the surface stress, we find

$$
L_{t}=\iint_{z=0} \boldsymbol{u} \cdot \boldsymbol{F}_{s} \mathrm{~d} x \mathrm{~d} y=-\iint_{z=0} u u_{z}+v v_{z} \mathrm{~d} x \mathrm{~d} y .
$$

We now use a standard identity for Stokes flow by extending the surface to a large closed hemisphere and noting that the velocity vanishes far from the domain. The divergence theorem yields the standard result (cf. Aris 1990; Lubensky \& Goldstein 1996) that

$$
L_{t}=-\frac{1}{2} \iiint_{z<0}\left|e_{i j}\right|^{2} \mathrm{~d} x \mathrm{~d} y \mathrm{~d} z,
$$

where $e_{i j}$ is the rate-of-strain tensor

$$
e_{i j}=\frac{1}{2}\left(\frac{\partial u_{i}}{\partial x_{j}}+\frac{\partial u_{i}}{\partial x_{j}}\right),
$$

by which we see that the arclength of the boundary decreases monotonically, unless the fluid is at rest. The action of the flow is to minimize the length of the boundary while preserving the area of the domain; the isoperimetric inequality suggests that, for domains of finite area, $\Omega$ will relax to a circle, or possibly the union of multiple circular domains. If we allow the domain to be infinite, a half-plane or an infinite strip could also be a (possibly local) energy minimizer.

\section{Stability and relaxation to a circular domain}

In this section we consider the relaxation of a linear perturbation to the boundary of a circular domain $\Omega$ of radius $R$. We know from the previous section that a circular domain is stable. However, rates of relaxation of the various modes are characteristic and give one possible way of measuring line tension (Mann et al. 1995). We expand the perturbation in Fourier modes, substitute in the governing equations, and retain each mode to first order in the perturbation.

We describe the boundary of the domain in polar coordinates as

$$
\mathscr{R}(\theta, t)=R+\epsilon \beta(\theta, t) .
$$

Rotational symmetry guarantees that angular Fourier modes will decouple in the linear stability problem. Moreover, area conservation of the domain requires that the $n=0$ mode vanishes. Consequently, we expand $\beta$ in the form

$$
\beta(\theta, t)=\sum_{n=1}^{\infty}\left[a_{n} \cos (n \theta)+b_{n} \sin (n \theta)\right] \mathrm{e}^{\lambda_{n} t}
$$

and compute to first order in $\epsilon$. 
Linearizing the curvature operator using standard identities, we find

$$
\begin{aligned}
\kappa(\mathscr{R}) & =-\frac{1}{R}+\epsilon \frac{1}{R^{2}}\left(\beta_{\theta \theta}+\beta\right)+O\left(\epsilon^{2}\right), \\
\kappa_{s}(\mathscr{R}) & =\frac{\epsilon}{R^{3}}\left(\beta_{\theta \theta \theta}+\beta_{\theta}\right)+O\left(\epsilon^{2}\right),
\end{aligned}
$$

and thus

$$
\kappa_{s}(\mathscr{R})=\epsilon \sum_{n=1}^{\infty}\left[-a_{n} \sin (n \theta)+b_{n}(t) \cos (n \theta)\right] \frac{n\left(1-n^{2}\right)}{R^{3}} \mathrm{e}^{\lambda_{n} t}
$$

to first order in $\epsilon$. We can now solve for the linearized surface-stress streamfunction; let

$$
S(r, \theta, t)=\epsilon \sum_{n=1}\left[a_{n} \sin (n \theta)-b_{n} \cos (n \theta)\right] \frac{n\left(1-n^{2}\right)}{R^{3}} \mathrm{e}^{\lambda_{n} t} s_{n}(r) .
$$

Substituting into Laplace's equation (2.41) for the surface stress yields

$$
\mathscr{L}_{r} s_{n}(r) \equiv\left(\frac{\partial^{2}}{\partial r^{2}}+\frac{1}{r} \frac{\partial}{\partial r}-n^{2}\right) s_{n}(r)=\delta(r-R),
$$

where $s_{n}$ must be regular at the origin and vanish in the far field. Note that at leading order the $\delta$-function forcing can be applied at the unperturbed boundary $(\mathscr{R}=R)$. Solving for $s_{n}(r)$, we find

$$
s_{n}(r)= \begin{cases}-R / 2 n(r / R)^{n}, & r<R \\ -R / 2 n(R / r)^{n}, & r>R\end{cases}
$$

To solve for the subfluid streamfunction, let

$$
\psi(r, \theta, z, t)=-\epsilon \sum_{n=1}^{\infty}\left[a_{n} \sin (n \theta)-b_{n} \cos (n \theta)\right] \mathrm{e}^{\lambda_{n} t} \frac{\left(1-n^{2}\right)}{2 R^{2}} P_{n}(r, z),
$$

where $P_{n}(r, z)$ satisfies Laplace's equation (2.39) in the subfluid:

$$
\begin{gathered}
\left(\mathscr{L}_{r}+\frac{\partial^{2}}{\partial z^{2}}\right) P_{n}(r, z)=0, \\
\frac{\partial P_{n}}{\partial z}(r, 0)=f_{n}(r) \equiv \begin{cases}(r / R)^{n}, & r<R, \\
(R / r)^{n}, & r>R,\end{cases}
\end{gathered}
$$

and $P_{n} \rightarrow 0$ for $r \rightarrow \infty$ and $z \rightarrow-\infty$. Once again as we are only retaining linear perturbations we may evaluate the surface-stress boundary condition for the circular domain, ignoring the deformation of the boundary.

As solutions to Laplace's equation in the subfluid take the form $J_{n}(k r) \mathrm{e}^{k z}$, we can construct the solution to the problem via a Hankel transform,

$$
P_{n}(r, z)=\int_{0}^{\infty} c_{n}(k) J_{n}(k r) \mathrm{e}^{k z} \mathrm{~d} k
$$

which implies

$$
\frac{\partial P_{n}}{\partial z}(r, 0)=\int_{0}^{\infty} c_{n}(k) J_{n}(k r) k \mathrm{~d} k=f_{n}(r) .
$$


Inverting the transform with the identity

$$
\delta\left(k-k^{\prime}\right)=\int_{0}^{\infty} k r J_{n}(k r) J_{n}\left(k^{\prime} r\right) \mathrm{d} r
$$

yields

$$
c_{n}(k)=\int_{0}^{\infty} f_{n}(r) J_{n}(k r) r \mathrm{~d} r
$$

or

$$
P_{n}(r, z)=\int_{0}^{\infty} \int_{0}^{\infty} f_{n}\left(r^{\prime}\right) J_{n}\left(k r^{\prime}\right) J_{n}(k r) \mathrm{e}^{k z} r^{\prime} \mathrm{d} r^{\prime} \mathrm{d} k .
$$

To complete the problem, we use the kinematic condition to advect the domain boundary. We linearize around $\mathscr{R}=R$,

$$
\mathscr{R}_{t}=\epsilon \beta_{t}=-\frac{1}{R} \psi_{\theta}(R, \theta) .
$$

Substituting the expressions (4.2) and (4.9) for $\beta$ and $\psi$ yields

$$
\begin{aligned}
\sum_{n=1}^{\infty}\left[a_{n} \cos (n \theta)+b_{n} \sin (n \theta)\right] & \lambda_{n} \mathrm{e}^{\lambda_{n} t} \\
& =\sum_{n=1}^{\infty}\left[a_{n} \cos (n \theta)+b_{n} \sin (n \theta)\right] \mathrm{e}^{\lambda_{n} t} \frac{n\left(1-n^{2}\right)}{2 R^{3}} P_{n}(R, 0),
\end{aligned}
$$

from which we deduce

$$
\lambda_{n}=\frac{n\left(1-n^{2}\right)}{2 R^{3}} P_{n}(R, 0)
$$

However,

$$
\begin{aligned}
P_{n}(R, 0) & =\int_{0}^{\infty} \int_{0}^{\infty} f_{n}\left(r^{\prime}\right) J_{n}\left(k r^{\prime}\right) J_{n}(k R) r^{\prime} \mathrm{d} r^{\prime} \mathrm{d} k \\
& =R \int_{0}^{\infty}\left[\int_{0}^{1}(\rho)^{n+1} J_{n}(k \rho)+\int_{1}^{\infty}(\rho)^{-n+1} J_{n}(k \rho) d \rho\right] J_{n}(k) \mathrm{d} k \\
& =2 n R \int_{0}^{\infty}\left[J_{n}(k)\right]^{2} \frac{\mathrm{d} k}{k^{2}} \\
& =\frac{2 n R}{\pi\left(n^{2}-\frac{1}{4}\right)},
\end{aligned}
$$

which allows a closed form for the relaxation rates,

$$
\lambda_{n}=-\frac{n^{2}\left(n^{2}-1\right)}{\pi R^{2}\left(n^{2}-\frac{1}{4}\right)}, \quad \text { for } \quad n=1,2,3 \ldots
$$

This result can also be deduced from Stone \& McConnell (1995) as reported in Mann et al. (1995) where it was used to estimate the line tension from the relaxation rate of perturbations to a circular domain. Note that $\lambda_{1}=0$ corresponds to the translation symmetry of the domain, and $\lambda_{n}<0$ for $n \geqslant 2$, as expected, confirming that the circular domain is stable. 


\section{Linear stability of a tether}

The length of a tether between the two bola of a deformed domain $\Omega$ (see figure 1) can be orders of magnitude longer than wide. It can thus be reasonably approximated by a two-dimensional infinite strip in the surface of the subfluid. In this section we consider the relaxation of linear perturbations of an infinite strip of width $2 d$. Consider a domain occupying the region $-d \leqslant y \leqslant d$. We consider two classes of linear perturbations: varicose perturbations for which the domain is symmetric around the line $y=0$ and sinuous instabilities for which the perturbations are antisymmetric. The analysis is analogous to that of the previous section - expanding the perturbations in Fourier modes and solving to first order in the perturbation. The growth rates for the sinuous perturbation can be deduced from the results of DeKoker \& McConnell (1996) who analysed stripe patterns in an analogous lipid system.

For the varicose case, we assume the domain takes the form

$$
|y|<H(x, t)=d+\epsilon h(x, t) .
$$

Translational symmetry guarantees that Fourier modes in $x$ decouple. Consequently, we expand $h(x, t)$ in the form

$$
h(x, t)=\int_{-\infty}^{\infty} \hat{h}(k) \mathrm{e}^{\mathrm{i} k x+\lambda_{k} t} \mathrm{~d} k .
$$

We linearize the curvature operator; using standard identities, we find that on the upper boundary

$$
\begin{aligned}
\kappa & =\epsilon h_{x x}+O\left(\epsilon^{2}\right), \\
\kappa_{s} & =-\epsilon h_{x x x}+O\left(\epsilon^{2}\right),
\end{aligned}
$$

from which we derive

$$
\kappa_{s}=\epsilon \int_{-\infty}^{\infty} \mathrm{i} k^{3} \hat{h}(k) \mathrm{e}^{i k x+\lambda_{k} t} \mathrm{~d} k,
$$

where we have dropped terms of order $\epsilon^{2}$ and higher. To solve for the linearized surface-stress streamfunction, let

$$
S(x, y, t)=\epsilon \int_{-\infty}^{\infty} \mathrm{i} k^{3} \hat{S}(k, y) \mathrm{e}^{i k x+\lambda_{k} t} \mathrm{~d} k .
$$

Laplace's equation (2.41) for the surface stress implies

$$
\left(\frac{\partial^{2}}{\partial y^{2}}-k^{2}\right) \hat{S}(k, y)=-[\delta(y-d)-\delta(y+d)]
$$

with $|\hat{S}(k, y)| \rightarrow 0$ as $|y| \rightarrow \infty$. Solving,

$$
\hat{S}(k, y)= \begin{cases}\left(\mathrm{e}^{-|k| y} / k\right) \sinh (k h), & d \leqslant y, \\ \left(\mathrm{e}^{-|k| d} / k\right) \sinh (k y), & |y|<d, \\ -\left(\mathrm{e}^{|k| y} / k\right) \sinh (k h), & y \leqslant-d .\end{cases}
$$

We now solve for the subfluid streamfunction; let

$$
\psi(x, y, z, t)=\epsilon \int_{-\infty}^{\infty} \mathrm{i} k^{2} \hat{P}(k, y, z) \mathrm{e}^{\mathrm{i} k x+\lambda_{k} t} \mathrm{~d} k,
$$


where $\hat{P}(k, y, z)$ must satisfy Laplace's equation (2.39) in the subfluid:

$$
\begin{aligned}
& \left(\frac{\partial^{2}}{\partial y^{2}}+\frac{\partial^{2}}{\partial z^{2}}-k^{2}\right) \hat{P}(k, y, z)=0, \quad z<0, \\
& \frac{\partial \hat{P}}{\partial z}(k, y, 0)=f(k, y) \equiv \begin{cases}\mathrm{e}^{-|k| y} \sinh (k h), & d \leqslant y, \\
\mathrm{e}^{-|k| d} \sinh (k y), & |y|<d, \\
-\mathrm{e}^{|k| y} \sinh (k h), & y \leqslant-d .\end{cases}
\end{aligned}
$$

and $\hat{P}(k, y, z)$ vanishes as $z \rightarrow-\infty$. Note that as we are only retaining linear perturbations we may evaluate the surface-stress boundary condition at $z= \pm d$, ignoring the perturbations to the boundary.

Solutions to Laplace's equation in the subfluid take the form $\exp \left(\mathrm{i} \ell y+\sqrt{k^{2}+\ell^{2}} z\right)$, so we construct the general solution to the problem:

$$
\hat{P}(k, y, z)=\int_{-\infty}^{\infty} c(k, \ell) \mathrm{e}^{\mathrm{i} \ell y+\sqrt{k^{2}+\ell^{2} z}} \mathrm{~d} \ell .
$$

Thus

$$
\frac{\partial \hat{P}}{\partial z}(k, y, 0)=\int_{-\infty}^{\infty} \sqrt{k^{2}+\ell^{2}} c(k, \ell) \mathrm{e}^{\mathrm{i} \ell y} \mathrm{~d} \ell=f(k, y) .
$$

Inverting the Fourier transform yields

$$
c(k, \ell)=\frac{1}{2 \pi \sqrt{k^{2}+\ell^{2}}} \int_{-\infty}^{\infty} f(k, y) \mathrm{e}^{-\mathrm{i} \ell y} \mathrm{~d} y .
$$

To complete the problem, we use the kinematic condition to advect the domain boundary. Linearizing around $z=d$ we find

$$
H_{t}=\epsilon h_{t}=-\psi_{x}(x, d, 0) \text {. }
$$

Substituting the expressions (5.2) and (5.9) for $h$ and $\psi$, we find

$$
\int_{\infty}^{\infty} \lambda_{k} \hat{h}(k) \mathrm{e}^{\mathrm{i} k x+\lambda_{k} t} \mathrm{~d} k=-\int_{-\infty}^{\infty} k^{3} \hat{P}(k, d, 0) \mathrm{e}^{\mathrm{i} k x+\lambda_{k} t} \mathrm{~d} k,
$$

from which we deduce

$$
\lambda_{k}=-k^{3} \hat{P}(k, d, 0)
$$

However

$$
\begin{aligned}
\hat{P}(k, d, 0) & =\frac{1}{2 \pi} \int_{-\infty}^{\infty} \frac{\mathrm{e}^{\mathrm{i} \ell d}}{\sqrt{k^{2}+\ell^{2}}} \int_{-\infty}^{\infty} f\left(k, y^{\prime}\right) \mathrm{e}^{-\mathrm{i} \ell y^{\prime}} \mathrm{d} y^{\prime} \mathrm{d} \ell \\
& =\int_{-\infty}^{\infty} f\left(k, y^{\prime}\right)\left[\frac{1}{2 \pi} \int_{-\infty}^{\infty} \frac{\mathrm{e}^{-\mathrm{i} \ell\left(y^{\prime}-d\right)}}{\sqrt{k^{2}+\ell^{2}}} \mathrm{~d} \ell\right] \mathrm{d} y^{\prime} \\
& =\frac{1}{\pi} \int_{-\infty}^{\infty} f\left(k, y^{\prime}\right) K_{0}\left(\left|k\left(d-y^{\prime}\right)\right|\right) \mathrm{d} y^{\prime},
\end{aligned}
$$

from which we deduce

where

$$
\lambda_{k}=-\frac{k^{2}}{\pi} I_{v}(|k d|)
$$

$$
I_{v}(\alpha)=\alpha \int_{-\infty}^{\infty} F(\alpha, \xi) K_{0}(\alpha|1-\xi|) \mathrm{d} \xi
$$


and

$$
F(\alpha, \xi) \equiv \begin{cases}\mathrm{e}^{-\alpha \xi} \sinh (\alpha), & 1 \leqslant \xi \\ \mathrm{e}^{-\alpha} \sinh (\alpha \xi), & |\xi|<1 \\ -\mathrm{e}^{\alpha \xi} \sinh (\alpha), & \xi \leqslant-1\end{cases}
$$

A laborious calculation reduces this to

$$
I_{v}(\alpha)=1-\cosh (2 \alpha)+\int_{0}^{2 \alpha} \sinh (2 \alpha-\zeta) K_{0}(\zeta) \mathrm{d} \zeta=1-2 \alpha K_{1}(2 \alpha)
$$

Note for $\alpha \ll 1$, we find

$$
I_{v}(\alpha)=\alpha^{2}(1-2 \gamma-2 \ln (\alpha))+\alpha^{4}\left(\frac{5}{4}-\gamma-\ln (\alpha)\right)+O\left(\alpha^{6} \ln \alpha\right),
$$

where $\gamma$ denotes Euler's constant. Also, as is clear from figure 3, $\left|I_{v}(\alpha)\right|<1$ and as $\alpha \rightarrow \infty$, we see that $I_{v}(\alpha)$ increases monotonically to 1 .

For the sinuous case, we will assume the perturbations to the domain are antisymmetric at leading order,

$$
-d+\epsilon h(x, t)<y<d+\epsilon h(x, t) .
$$

We again expand $h(x, t)$ in the form

$$
h(x, t)=\int_{-\infty}^{\infty} \hat{h}(k) \mathrm{e}^{\mathrm{i} k x+\lambda_{k} t} \mathrm{~d} k .
$$

A similar calculation yields

$$
\begin{gathered}
\lambda_{k}=-\frac{k^{2}}{\pi} I_{s}(|k d|), \\
I_{s}(\alpha)=\alpha \int_{-\infty}^{\infty} G(\alpha, \xi) K_{0}(\alpha|1-\xi|) \mathrm{d} \xi,
\end{gathered}
$$

and

$$
G(\alpha, \xi) \equiv \begin{cases}\mathrm{e}^{-\alpha \xi} \cosh (\alpha), & 1 \leqslant \xi \\ \mathrm{e}^{-\alpha} \cosh (\alpha \xi), & |\xi|<1 \\ \mathrm{e}^{\alpha \xi} \cosh (\alpha), & \xi \leqslant-1\end{cases}
$$

We find after some calculation that

$$
I_{s}(\alpha)=2-I_{v}(\alpha)=1+2 \alpha K_{1}(2 \alpha) .
$$

Consequently, for $\alpha \ll 1$, we find

$$
I_{s}(\alpha)=2+\alpha^{2}(2 \gamma-1+2 \ln (\alpha))+\alpha^{4}\left(\gamma-\frac{5}{4}+\ln (\alpha)\right)+O\left(\alpha^{6} \ln \alpha\right),
$$

where $\gamma$ again denotes Euler's constant. As $\alpha$ increases from $0, I_{s}(\alpha)$ decreases monotonically from 2 to 1 .

In the paper by DeKoker \& McConnell (1996), the drag a sinuous perturbation feels due to the subfluid is computed, and a simple calculation yields the decay rate computed above. Moreover, by interpreting these results as a balance between the drag and the line tension forcing, and noting that the subfluid drag is linear, we can deduce that averaging a sinuous and varicose perturbation is equivalent to perturbing the edge of a half-plane sinusoidally. This yields the result

$$
\frac{1}{2}\left[I_{s}(\alpha)+I_{v}(\alpha)\right]=1,
$$

where the 1 on the right-hand side arises from the half-plane problem which is equivalent to the limit of large $\alpha$ in both the sinuous and varicose problems. 


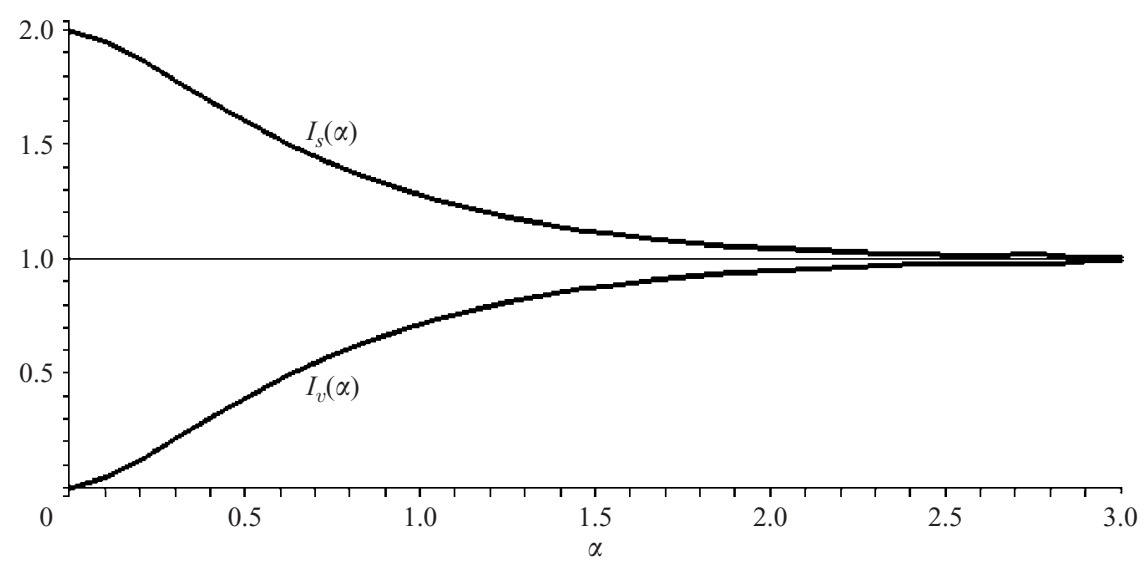

FIgURE 3. Growth factors for varicose and sinuous instabilities. Perturbations of wavelength $k$ to a strip of width $2 d$ decay at a rate $\lambda=-\left(k^{2} / \pi\right) I(\alpha)$ where $\alpha=k d$ and $I(\alpha)=I_{v}(\alpha)$ for varicose instabilities and $I(\alpha)=I_{s}(\alpha)$ for sinuous instabilities. We see that all instabilities are decaying indicating that the strip is stable to linear perturbations.

From the calculations above, we conclude that the tether is linearly stable, with sinuous perturbations damped more strongly than varicose variations of the same wavelength. Also, as the wavelength decreases to zero, we discover that the varicose perturbation decays at a rate $\lambda_{k} \sim-(2 / \pi) k^{4} d^{2} \ln (1 /(k d))$. It is tempting to assume that long-wave tether variations will lend themselves to analysis via lubrication theory in a fashion analogous to the Hele-Shaw problem (Almgren 1996). However, the logarithm in the long-wave growth rate foreshadows a difficulty: the lubrication theory is in fact non-local and contains an integral convolution term at leading order. In fact, it appears (to us) simpler and more productive to analyse the full problem via a boundary integral method as described in the next section.

\section{Boundary integral formulation}

Boundary integral formulations of free boundary problems often yield efficient numerical methods for describing their evolution. We note that for the ILLSS model the surface-stress streamfunction and in turn the subfluid streamfunction and fluid velocity are determined solely by the position of the domain boundary. Moreover, they are determined as a linear response to the forcing associated with the line tension. Consequently, the problem allows a boundary integral formulation where the velocity of the boundary of the domain can be determined as an integral over the boundary.

We first derive an integral formulation for the surface-stress streamfunction, which satisfies the Poisson equation

$$
\begin{aligned}
\nabla_{\perp}^{2} S & =-\kappa_{s} \delta(d) \\
& =-\oint \kappa_{s}\left(s^{\prime}\right) \delta\left(s^{\prime}\right) \delta(d) \mathrm{d} s^{\prime} .
\end{aligned}
$$

Note that the Green's function $G_{2 D}(r)$ for Laplace's equation in two dimensions satisfies

$$
\nabla_{\perp}^{2} G_{2 D}=\delta(x) \delta(y)
$$


which yields

$$
G_{2 D}(r)=\frac{1}{2 \pi} \ln r, \quad r=\sqrt{x^{2}+y^{2}} .
$$

As $(s, d)$ are orthogonal coordinates at the boundary of the domain, we can construct a solution for the surface-stress streamfunction in terms of the Green's function,

$$
\begin{aligned}
S(x, y) & =-\oint \kappa_{s}\left(s^{\prime}\right) G_{2 D}\left(\left|\boldsymbol{R}-\boldsymbol{\Gamma}\left(s^{\prime}\right)\right|\right) \mathrm{d} s^{\prime}, \quad \boldsymbol{R}=x \hat{\boldsymbol{\imath}}+y \hat{\boldsymbol{\jmath}}, \\
& =-\frac{1}{4 \pi} \oint \kappa_{s}\left(s^{\prime}\right) \ln \left|\boldsymbol{R}-\boldsymbol{\Gamma}\left(s^{\prime}\right)\right|^{2} \mathrm{~d} s^{\prime} \\
& =-\frac{1}{2 \pi} \oint \kappa\left(s^{\prime}\right) \frac{\left[\boldsymbol{\Gamma}_{s}\left(s^{\prime}\right)\right] \cdot\left[\boldsymbol{R}-\boldsymbol{\Gamma}\left(s^{\prime}\right)\right]}{\left|\boldsymbol{R}-\boldsymbol{\Gamma}\left(s^{\prime}\right)\right|^{2}} \mathrm{~d} s^{\prime},
\end{aligned}
$$

where the last step is derived via integration by parts.

This can be simplified by introducing a vector, $\boldsymbol{q}$, in the surface from a point on the boundary of the domain $(\boldsymbol{\Gamma})$ to an arbitrary point the surface $(\boldsymbol{R})$,

$$
\boldsymbol{q}(s)=\boldsymbol{R}-\boldsymbol{\Gamma}(s), \quad q=|\boldsymbol{q}|, \quad \hat{\boldsymbol{q}}=\frac{\boldsymbol{q}}{q},
$$

which allows us to write the solution for the surface-stress streamfunction as

$$
S(x, y)=-\frac{1}{2 \pi} \oint \kappa \hat{\boldsymbol{t}} \cdot \frac{\hat{\boldsymbol{q}}}{q} \mathrm{~d} s,
$$

where we have used the fact that $\boldsymbol{\Gamma}_{s}$ is the unit tangent $\hat{\boldsymbol{t}}$.

The subfluid streamfunction $\psi(x, y, z)$ can now be solved for in terms of $S(x, y)$; we know the solution is harmonic with a Neumann condition at the surface,

$$
\begin{aligned}
\nabla^{2} \psi & =0, \quad z<0, \\
\psi_{z} & =S(x, y), \quad z=0,
\end{aligned}
$$

and the fluid speed $|\nabla \psi|$ vanishes as $z \rightarrow-\infty$.

The associated Green's function $G_{3 D}(x, y, z)$, which satisfies

$$
\begin{aligned}
\nabla^{2} G_{3 D} & =0, \quad z<0, \\
\partial_{z}\left(G_{3 D}\right) & =\delta(x) \delta(y), \quad z=0,
\end{aligned}
$$

is easily derived, namely,

$$
G_{3 D}(x, y, z)=\frac{1}{2 \pi} \frac{1}{\sqrt{z^{2}+r^{2}}}, \quad r=\sqrt{x^{2}+y^{2}},
$$

which in turn implies

$$
\psi(x, y, z)=\int_{-\infty}^{\infty} \int_{-\infty}^{\infty} S\left(x^{\prime}, y^{\prime}\right) G_{3 D}\left(x-x^{\prime}, y-y^{\prime}, z\right) \mathrm{d} x^{\prime} \mathrm{d} y^{\prime} .
$$

It is now straightforward, if algebraically cumbersome, to substitute the expression for the surface-stress streamfunction into this integral formulation (evaluated at the surface), and exchange the order of integration to derive the streamfunction evaluated 
at the surface. We see that

$$
\begin{aligned}
\psi(x, y, 0) & =\int_{-\infty}^{\infty} \int_{-\infty}^{\infty} S\left(x^{\prime}, y^{\prime}\right) G_{3 D}\left(x-x^{\prime}, y-y^{\prime}, 0\right) \mathrm{d} x^{\prime} \mathrm{d} y^{\prime} \\
& =-\frac{1}{2 \pi} \int_{-\infty}^{\infty} \int_{-\infty}^{\infty}\left[\oint \kappa \hat{t} \cdot \frac{\hat{q}}{q} \mathrm{~d} s\right] G_{3 D}\left(x-x^{\prime}, y-y^{\prime}, 0\right) \mathrm{d} x^{\prime} \mathrm{d} y^{\prime} \\
& =\oint \kappa \hat{t} \cdot \boldsymbol{K}(\boldsymbol{\Gamma}(s), \boldsymbol{R}) \mathrm{d} s
\end{aligned}
$$

where

$$
\begin{aligned}
\boldsymbol{K}\left(\boldsymbol{R}^{\prime}, \boldsymbol{R}^{\prime \prime}\right) & =-\frac{1}{(2 \pi)^{2}} \int_{-\infty}^{\infty} \int_{-\infty}^{\infty} \frac{\boldsymbol{R}^{\prime}-\boldsymbol{R}}{\left|\boldsymbol{R}^{\prime}-\boldsymbol{R}\right|^{2}\left|\boldsymbol{R}^{\prime \prime}-\boldsymbol{R}\right|} \mathrm{d} x \mathrm{~d} y \\
& =\frac{1}{2 \pi} \frac{\boldsymbol{R}^{\prime}-\boldsymbol{R}^{\prime \prime}}{\left|\boldsymbol{R}^{\prime}-\boldsymbol{R}^{\prime \prime}\right|} .
\end{aligned}
$$

The last integral above can be evaluated by changing to polar coordinates with origin at $\boldsymbol{R}^{\prime}$ and with $\boldsymbol{R}^{\prime \prime}$ along the polar axis, evaluating the radial integral on a finite disk centred at the origin, evaluating the angular integral and then letting the radius approach infinity. Consequently, we conclude that

$$
\psi(x, y)=-\frac{1}{2 \pi} \oint \kappa \hat{\boldsymbol{t}} \cdot \hat{\boldsymbol{q}} \mathrm{d} s, \quad \hat{q}=\frac{\boldsymbol{R}-\boldsymbol{\Gamma}(s)}{|\boldsymbol{R}-\boldsymbol{\Gamma}(s)|}, \quad \boldsymbol{R}=x \hat{\boldsymbol{\imath}}+y \hat{\boldsymbol{\jmath}} .
$$

To finish the formulation we must specify the motion of the boundary; from the kinematic condition (2.42) we know

$$
\frac{\mathrm{D} \boldsymbol{\Gamma}}{\mathrm{D} t}=\left.\left(\boldsymbol{U}+\boldsymbol{U}_{\mathrm{ext}}\right)\right|_{\partial \Omega}=\left.\left(\hat{\boldsymbol{k}} \times \nabla_{\perp} \psi+\boldsymbol{U}_{\mathrm{ext}}\right)\right|_{\partial \Omega} .
$$

At this point we turn our attention from the physical calculation to setting up the numerical calculation of the boundary integral formulation. We first parameterize the boundary, $\boldsymbol{\Gamma}(p, t)$, with a parameter $p$ which is $2 \pi$-periodic. We then note that as the boundary is isotropic, we are free to introduce an arbitrary tangential velocity whose only effect will be to change the parameterization of the boundary (cf. Hou et al. 1994). Consequently, the tangential component, $\mathscr{V}$, can be chosen arbitrarily (and in fact will be used to distribute the collocation points evenly on the boundary). So an equivalent formulation is

$$
\boldsymbol{\Gamma}_{t}=\left(\mathscr{U}+\mathscr{U}_{\mathrm{ext}}\right) \hat{\boldsymbol{n}}+\mathscr{V} \hat{\boldsymbol{t}},
$$

where

$$
\begin{gathered}
\mathscr{U}_{\mathrm{ext}}=\hat{\boldsymbol{n}} \cdot \boldsymbol{U}_{\mathrm{ext}}, \\
\mathscr{\boldsymbol { U }}=\hat{\boldsymbol{n}} \cdot \hat{\boldsymbol{k}} \times\left.\nabla_{\perp} \psi\right|_{\partial \Omega}=\left.\psi_{s}\right|_{\partial \Omega},
\end{gathered}
$$

and $\mathscr{V}$ is arbitrary. It now suffices to know the streamfunction only on the boundary of the domain; this simplifies our numerical calculations immensely.

In summary, we find that

$$
\boldsymbol{\Gamma}_{t}=\left(\Psi_{s}+\mathscr{U}_{\mathrm{ext}}\right) \hat{\boldsymbol{n}}+\mathscr{V} \hat{\boldsymbol{t}},
$$

where $\Psi(s)$ is the streamfunction restricted to the boundary of the domain,

$$
\Psi(s)=-\frac{1}{2 \pi} \oint \kappa \hat{\boldsymbol{t}} \cdot \hat{\boldsymbol{Q}} \mathrm{d} s^{\prime}, \quad \hat{\boldsymbol{Q}}=\frac{\boldsymbol{\Gamma}\left(s^{\prime}\right)-\boldsymbol{\Gamma}(s)}{\left|\boldsymbol{\Gamma}\left(s^{\prime}\right)-\boldsymbol{\Gamma}(s)\right|},
$$


and $\mathscr{V}$ can be chosen arbitrarily. Once the externally imposed velocity $\boldsymbol{U}_{\text {ext }}$ and the initial domain boundary location have been specified, the boundary integral equations (6.21), (6.22) completely determine the evolution of the Langmuir layer.

\subsection{Boundary integral numerics}

We report on a continuing effort to numerically simulate the boundary integral equations (6.21), (6.22). Our method is based on the work of Hou et al. (1994) who recognized the importance of using an intrinsic description of the boundary which allows an accurate implicit solution for the high-wavenumber modes avoiding numerical instabilities. Figures 4, 5 illustrate that such a method reproduces the observed bola dynamics qualitatively; we expect to report a quantitative comparison elsewhere after we have refined our numerical simulations and experimental technique.

Previously a boundary integral formulation for the Langmuir layer (including electrostatic forces) was proposed by Lubensky \& Goldstein (1996) and implemented by Heinig et al. (2004); they compute the boundary velocity explicitly, which is equivalent to computing $\hat{\boldsymbol{k}} \times \nabla_{\perp} \psi$ in (6.16), (6.17). Heinig et al. (2004) were able to qualitatively reproduce many experimental results, although the scheme exhibited moderate area loss. Moreover, their scheme was explicit and first-order in time which limits the size of time-steps that can be taken accurately. Here we present a scheme that is second-order in time, essentially spectrally accurate in space, and semi-implicit which guarantees stability. Moreover, by using the conservative form of the boundary integral equations $(6.21),(6.22)$ where the velocity is an exact derivative with respect to arclength our scheme better conserves the area of the domain.

Following Hou et al. (1994), we represent the boundary with an equal-arclength discretization; note that this equal-arclength constraint specifies the tangential velocity. Derivatives are computed pseudo-spectrally (Gottlieb \& Orszag 1977; Trefethen 2000), and the boundary integral is computed using either Romberg integration or a 16-panel closed Newton-Cotes formula which guarantees high-order spatial accuracy. Numerically we see that the problem is extremely stiff and explicit integration methods are highly susceptible to high-wavenumber instabilities. This can be ameliorated by operator splitting following the ideas of Hou et al. (1994). While such a splitting is not immediately apparent in the formulation above, the formulation in Lubensky \& Goldstein (1996) and Heinig et al. (2004) can be used to show that asymptotically the high wavenumbers are governed by a much simpler evolution law, namely motion by mean curvature. It is straightforward to solve the evolution by mean curvature implicitly and to high accuracy (cf. Hou et al. 1994). We proceed by using Strang splitting with the mean-curvature step implemented implicitly and the external velocity and the boundary integral velocity minus the mean curvature velocity computed explicitly.

We implemented this algorithm using MATLAB. Numerically, we found that it was necessary to correct the arclength discretization regularly to correct a slow drift of the grid points - this was done using spectral interpolation and a Newton-Raphson iteration. Also, it is necessary to filter the highest wavenumber modes in the boundary integral (whose numerical accuracy is poor anyway due to the discretization); in practice we convolute the spectrum with a smooth filter and retain roughly two-thirds of the spectrum. Details of the numerical implementation can be found in Pugh (2006).

The specific numerical integration illustrated in figures 4, 5 uses 1024 points and 64 time-steps per unit of time. The domain initially is a circle of radius 3 . An external straining flow $\boldsymbol{U}_{\text {ext }}=0.25(x \hat{\boldsymbol{\imath}}-y \hat{\boldsymbol{J}})$ is imposed and the domain is stretched into a 


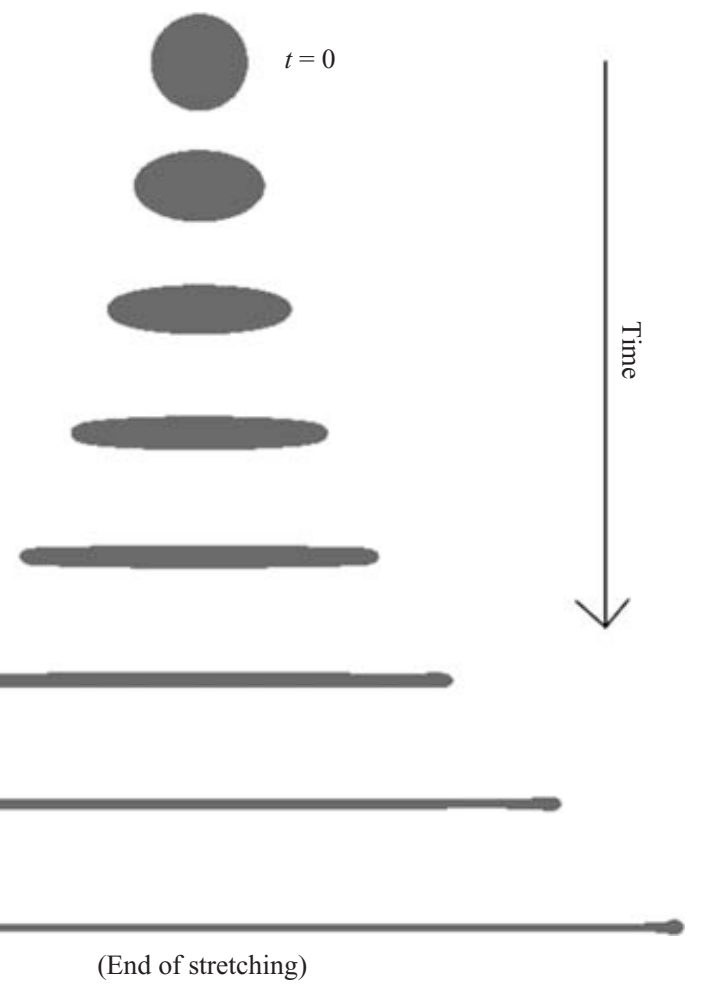

FIGURE 4. A series of snapshots of a numerical evolution of the stretching of a Langmuir domain computed via a boundary integral method for the inviscid Langmuir layer Stokesian subfluid Model. The domain is originally a circle of radius 3. At the end of the stretching the domain is 60 units long. The domain is stretched by a transient straining flow of strength 0.25 for 11.14 units of time; snapshots are separated by 1.59 units of time.

long narrow lozenge of length 60 with an aspect ratio of roughly 144-to-1. As the domain relaxes it loses convexity with the appearance of two rounded reservoirs at the tips connected by a narrow tether, creating the classic bola shape observed in the experiments. Eventually the domain regains convexity, becomes nearly elliptical, and relaxes towards the circular energy minimizer.

This numerical run took about thirty hours on a $\mathrm{GHz}$ speed single-processor machine. A check on the accuracy of the code is that the area of the domain should be conserved; during the stretching phase the domain loses roughly $1 \%$ of its area; during the relaxation phase the area is conserved to within $0.1 \%$.

\subsection{Computing the line tension}

In this section we show how to compute the line tension from the tether relaxation velocity. Experiments on bola relaxation have been used previously to make orderof-magnitude estimates of line tensions (Benvegnu \& McConnell 1992; Mann et al. 1992, 1995). Benvegnu \& McConnell (1992) modelled the bola as a circular disk being pulled by the line tension associated with the tether. By computing the force exerted by the line tension and balancing it with the drag on the disk they estimate the line tension. We summarize their calculation below; note that we have returned to the original dimensional variables. 


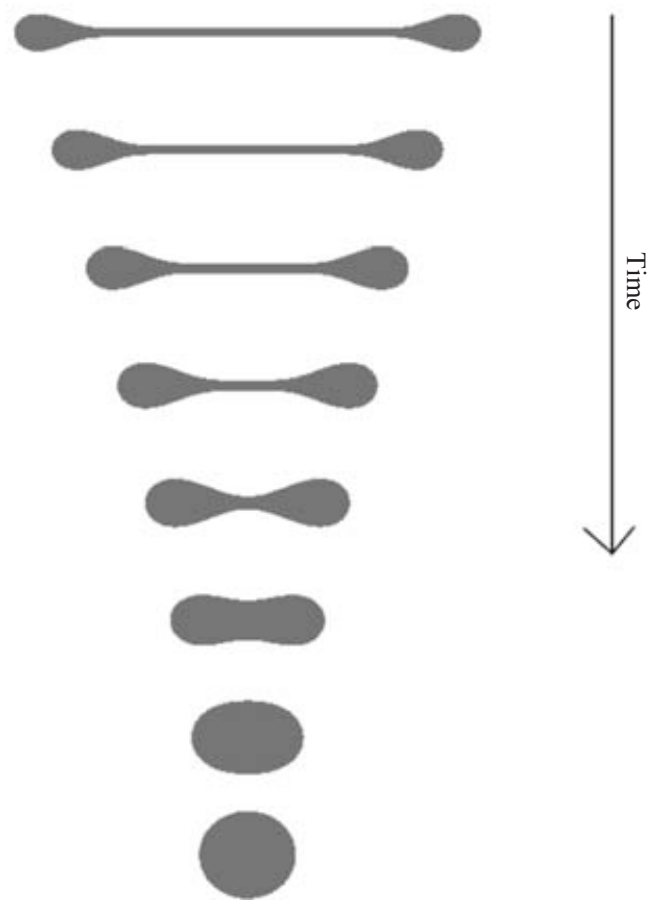

FIGURE 5. Tether relaxation. After the straining field is released in figure 4, the domain assumes the classic bola shape, and eventually relaxes back to an ellipse approaching the energy-minimizing circular configuration. Here the snapshots are separated by 13.1 units of time. A movie of this numerical evolution is available with the online version of the paper.

The total horizontal force on the disk, $\boldsymbol{F}_{\text {line }}$, is easily computed by integrating the force around one end of the tether. For definiteness, consider the right half of one the tethers in figure 5 and integrate along a contour $\mathscr{C}$ from the midpoint of the bottom of the tether counter-clockwise to the midpoint of the top of the tether,

$$
\boldsymbol{F}_{\text {line }}=\int_{\mathscr{C}} \lambda \kappa \hat{\boldsymbol{n}} \mathrm{d} s=\left.\lambda \hat{\boldsymbol{t}}\right|_{\text {bottom }} ^{\text {top }}=-2 \lambda \hat{\boldsymbol{j}},
$$

yielding a force proportional to the line tension in the direction of motion.

The drag force is the product of the bola velocity and the drag coefficient,

$$
\boldsymbol{F}_{\text {drag }}=C_{\text {drag }} V_{\text {bola }} \hat{\boldsymbol{J}} \text {. }
$$




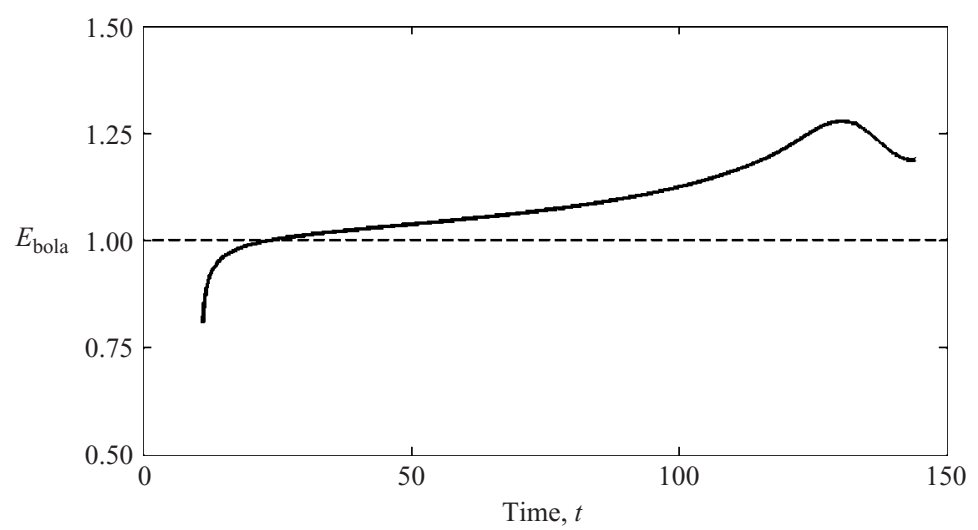

Figure 6. Computing line tension from the tether relaxation. Previous studies have assumed that the line tension for a relaxing tether can be computed from $\lambda=E_{\text {bola }} 4 \eta^{\prime} V_{\text {bola }} R_{\text {bola }}$ where $V_{\text {bola }}$ is the bola velocity and $R_{\text {bola }}$ is the tether radius, measured as the maximum half-width of the bola perpendicular to the tether. Here we plot $E_{\text {bola }}$ for the numerically computed relaxation in figure 5; the graph starts when the straining flow stops at $t=11.14$ and stops when the domain loses convexity at roughly $t=144.5$. Previous studies assumed that $E_{\text {bola }}$ was unity after the initial relaxation and before the two bolas merge. In fact it slowly increases from approximately 1 to a maximum of 1.3 in the regime where the ends of the bola are interacting. These results suggest that Mann et al. (1995) underestimates the line tension by perhaps $20 \%$.

The drag coefficient was approximated by Benvegnu \& McConnell (1992) in two ways: first as half the drag on a flat disk of radius $R_{\text {bola }}$ in an infinite fluid, which yields $C_{\text {drag }}=\frac{16}{3} \eta^{\prime} R_{\text {bola }}$ (cf. section 339, Lamb 1932); the second model considers the drag on a solid disk in an inviscid monolayer on an infinite subfluid, which yields $C_{\text {drag }}=8 \eta^{\prime} R_{\text {bola }}$ (cf. Hughes et al. 1981).

We can now solve for the line tension by equating $\boldsymbol{F}_{\text {line }}$ and $\boldsymbol{F}_{\text {drag }}$; this yields

$$
\lambda=E_{\text {bola }} 4 \eta^{\prime} V_{\text {bola }} R_{\text {bola }},
$$

where $E_{\text {bola }}=C_{\text {drag }} / 8$, which is $2 / 3$ for the first model and unity for the second model. Typically $R_{\text {bola }}$ is estimated as half the maximum width of the bola measured perpendicular to the tether axis.

Figure 6 allows us to estimate $E_{\text {bola }}$ from our numerical simulations; as we have non-dimensionalized the problem we can set the viscosity and line tension to unity to yield

$$
E_{\text {bola }}=\frac{1}{4 V_{\text {bola }} R_{\text {bola }}} .
$$

We assume that this model should be valid at times after the domain has relaxed to a bola shape and before the two ends of the bola have begun to interact. In fact we find that $E_{\text {bola }}$ rapidly increases to slightly above unity as the ends of the bola become bulbous. It then slowly increases to a maximum of roughly 1.3 where the two ends of the tether are clearly interacting. This suggests that choosing $C_{\mathrm{drag}}=8 \eta^{\prime} R_{\mathrm{bola}}$ is nearly correct and that the results of Mann et al. (1995) which use this approximation underestimate the line tension by perhaps $20 \%$. Our simulations also suggest that variations in bola length and tether thickness can cause changes in the relaxation rate of the same order; this effect is explored in Pugh (2006). 


\section{Discussion}

In this paper we have developed a model of a Langmuir layer with two fluid phases, one of which is localized into a compact domain. Dimensional analysis suggests that the dominant balance is between the driving line tension at the domain boundary and the viscous drag of the subfluid. The governing hydrodynamic equations have been reduced to a more tractable form: the inviscid Langmuir layer Stokesian subfluid (ILLSS) model discussed herein reduces the problem to solving for a horizontal streamfunction which is harmonic in the subfluid, and the surface-stress streamfunction which is harmonic in the Langmuir layer domains. A further reduction yields a boundary integral formulation which can be efficiently numerically integrated. The model conforms well to experimentally observed behaviour of Langmuir layers.

The dissipation of energy suggests that an isolated compact domain will evolve towards a circular equilibrium which minimizes its perimeter. It is also possible to compute the relaxation rates of perturbations of a circular domain, which has been an effective tool for estimating line tensions (Mann et al. 1992, 1995; Lauger et al. 1996). In particular the amplitude of the $n$th Fourier mode decays as $\exp \left(-t / \tau_{n}\right)$ where $\tau_{n}$ is the characteristic relaxation time,

$$
\tau_{n}=\frac{\pi R^{2} \eta^{\prime}}{\lambda} \frac{\left(n^{2}-\frac{1}{4}\right)}{n^{2}\left(n^{2}-1\right)}, \quad \text { for } \quad n=1,2,3 \ldots,
$$

where $\pi R^{2}$ is the domain area, $\eta^{\prime}$ is the subfluid viscosity and $\lambda$ is the line tension. These relaxation rates are deduced from (4.19) and agree with equation (A18) in Mann et al. (1995) which was based on the earlier work of Stone \& McConnell (1995).

We also consider perturbations of an infinite strip as a model of the narrow tether seen in the bola configuration. Linear theory indicates that the infinite strip is stable to perturbations, in agreement with the experimental observation of tethers. A logical next analytical step is to pursue a nonlinear lubrication theory model of long-wave perturbations to the tether; this is a strategy that works well, for example, for analysing rupture in the Hele-Shaw problem (Almgren 1996; Almgren et al. 1996; Constantin et al. 1993; Dupont et al. 1993; Goldstein et al. 1993). However, our analysis (not reported here) shows that the lubrication model for this problem is non-local and contains an integral term which makes the analysis quite complicated. A simpler alternative is to convert the problem to a boundary integral formulation and attack it numerically.

In the penultimate section of this paper we derive a boundary integral formulation for the ILLSS model which is capable of incorporating an external irrotational flow. Our numerical implementation of a circular patch in a stagnation-point flow shows that it is stretched into a long and narrow filament. When the stagnation flow is turned off, the domain first develops circular bulges at its ends, creating the characteristic bola shape. The bulbous ends then slowly migrate towards each other, eventually merging and relaxing to a circular domain, the ubiquitous energy minimizer.

Experiments on bola relaxation have been used previously to make order-ofmagnitude estimates of line tensions (Benvegnu \& McConnell 1992; Mann et al. 1992, 1995). However these estimates rely on heuristic theories and dimensional analysis; our numerical simulation suggest that Mann et al. (1995) underestimate line tensions by perhaps $20 \%$ which is similar to the reported experimental uncertainty.

We believe comparisons of the fully nonlinear numerical simulation to the experimental observations of bola relaxation will allow a more accurate determination 
of the line tension in a variety of systems. Unlike relaxation rates for perturbation of a circular domain, these measurements are not limited to the regime where linear theory is applicable. In conclusion, we believe that the ILLSS model effectively models many experimental observations of Langmuir layers while remaining analytically and numerically tractable.

The authors would like to thank the referees for their careful and cogent comments on an earlier draft of this paper. A.J.B. and J. R. W. would like to thank Harvey Mudd College for financial support and UCLA for summer support via NSF grants DMS-0535521 and ACI-0321917, and ONR grant N000140410078. L. Z. and E. K. M. were partially supported by the National Science Foundation under Grant No. DMR9984304.

\section{REFERENCES}

Adamson, A. W. \& Gast, A. P. 1998 Physical Chemistry of Surfaces, 6th Edn. John Wiley and Sons. Almgren, R. 1996 Singularity formation in Hele-Shaw bubbles. Phys. Fluids 8, 344-352.

Almgren, R., Bertozzi, A. \& Brenner, M. P. 1996 Stable and unstable singularities in the unforced Hele-Shaw cell. Phys. Fluids 8, 1356-1370.

ARIs, R. 1990 Vectors, Tensors, and the Basic Equations of Fluid Mechanics. Dover.

Benvegnu, D. J. \& McConnell, H. M. 1992 Line tension between liquid domains in lipid monolayers. J. Phys. Chem. 96, 6820-6824.

Brochard-Wyart, F. 1990 Stability of a liquid ribbon spread on a liquid surface. C. R. Acad. Sci Ii 311, 295-300.

Constantin, P., Dupont, T. F., Goldstein, R. E., Kadanoff, L. P., Shelley, M. J. \& Zhou, S.-M. 1993 Droplet breakup in a model of the Hele-Shaw cell. Phys. Rev. E 47, 4169-4181.

DeKoKer, R. \& McConnell, H. M. 1993 Circle to dogbone - shapes and shape transitions of lipid monolayer domains. J. Phys. Chem. 97, 13419-13424.

DeKoker, R. \& McConnell, H. M. 1996 Stripe phase hydrodynamics in lipid monolayers. J. Phys. Chem. 100, 7722-7728.

Drazin, P. G. \& Reid, W. H. 2004 Hydrodynamic Stability, 2nd Edn. Cambridge University Press.

Dupont, T. F., Goldstein, R. E., Kadanoff, L. P. \& Zhou, S.-M. 1993 Finite-time singularity formation in Hele-Shaw systems. Phys. Rev. E 47, 4182-4196.

EDIDIn, M. 2003 The state of lipid rafts: From model membranes to cells. Annu. Rev. Biophys. Biomol. Structure 32, 257-283.

GAINES JR, G. L. 1966 Insoluble Monolayers at Liquid-Gas Interfaces. Interscience.

Glasner, K. 2003 A diffuse interface approach to Hele-Shaw flow. Nonlinearity 16, 49-66.

Goldstein, R. E., Pesci, A. I. \& Shelley, M. J. 1993 Topology transitions and singularities in viscous flows. Phys. Rev. Lett. 70, 3043-3046.

Goodrich, F. C. 1981 The theory of capillary excess viscosities. Proc. R. Soc. Lond. A 374, 341-370.

Gottlieb, D. \& Orszag, S. A. 1977 Numerical Analysis of Spectral Methods: Theory and Applications. Society for Industrial and Applied Mathematics.

Heinig, P., Helseth, L. E. \& Fischer, T. M. 2004 Relaxation of patterns in 2D modulated phases. New J. Phys. 6, 189.

Hou, T., Lowengrub, J. \& Shelley, M. 1994 Removing the stiffness from interfacial flow with surface tension. J. Comput. Phys. 114, 312-338.

Hughes, B. D., Pailthorpe, B. A. \& White, L. R. 1981 The translational and rotational drag on a cylinder moving in a membrane. J. Fluid Mech. 110, 349-372.

JoLY, M. 1972 Rheological properties of monomolecular films: Part ii: Experimental results. theoretical interpretation. applications. Surface Colloid Sci. 5, 79-193.

Lamb, H. 1932 Hydrodynamics. Cambridge University Press.

Lauger, J., Robertson, C. R., Frank, C. W. \& Fuller, G. G. 1996 Deformation and relaxation processes of mono- and bilayer domains of liquid crystalline langmuir films on water. Langmuir 12 (23), 5630-5635. 
Lubensky, D. K. \& Goldstein, R. E. 1996 Hydrodynamics of monolayer domains at the air-water interface. Phys. Fluids 8, 843-854.

Lucassen, J., Akamatsu, S. \& Rondelez, F. 1991 Formation, evolution, and rheology of 2dimensional foams in spread monolayers at the air-water interface. J. Colloid Interface Sc. 144, 434-448.

ManN, E. K. 1992 PDMS films at water surfaces: texture and dynamics. PhD thesis, Université de Paris VI.

Mann, E. K., HÉnon, S. \& Langevin, D. 1992 Meunier molecular layers of a polymer at the free water surface: Microscopy at the Brewster angle. J. Phys. Paris 2, 1683-1704.

Mann, E. K., Hénon, S., Langevin, D., Meunier, J. \& Léger, L. 1995 The hydrodynamics of domain relaxation in a polymer monolayer. Phys. Rev. E 51, 5708-5720.

Mann, E. K. \& PrimaK, S. 1999 The stability of two-dimensional foams in Langmuir monolayers. Phys. Rev. Lett. 83, 5397-5400.

MANn JR, J. A. 1985 Dynamics, structure and function of interfacial regions. Langmuir 1, 10-23.

Mann JR, J. A., Crouser, P. D. \& Meyer, W. V. 2001 Surface fluctuation spectroscopy by surfacelight-scattering spectroscopy. Appl. Optics 40 (24), 4092-4112.

Mayor, S. \& RaO, M. 2004 Rafts: Scale-dependent, active lipid organization at the cell surface. Traffic 5, 231-240.

DE Mul, M. N. G. \& ManN JR, J. A. 1998 Determination of the thickness and optical properties of a langmuir film from the domain morphology by Brewster angle microscopy. Langmuir 14, 2455-2466.

Parton, R. G. \& Hancock, J. F. 2004 Lipid rafts and plasma membrane microorganization: Insights from Ras. Trends Cell Biol. 14 (3), 141-147.

Powers, T. R., Huber, G. \& Goldstein, R. E. 1990 Fluid-membrane tethers: Minimal surfaces and elastic boundary layers. Phys. Rev. E 65 (4), 041901.

PozRIKIDIS, C. 1992 Boundary Integral and Singularity Methods for Linearized Viscous Flow. Cambridge University Press.

Pugh, J. M. 2006 Numerical simulation of domain relaxation in Langmuir films. Senior Thesis, Department of Physics, Harvey Mudd College.

Simons, K. \& IKonen, E. 1997 Functional rafts in cell membranes. Nature 387, 569-572.

StefFen, P., Wurlitzer, S. \& Fischer, T. M. 2001 Hydrodynamics of shape relaxation in viscous langmuir monolayer domains. J. Phys. Chem. A 105 (36), 8281-8283.

Stone, H. A. \& McConnell, H. M. 1995 Hydrodynamics of quantized shape transitions of lipid domains. Proc. R. Soc. Lond. A 448, 97-111.

Trefethen, L. N. 2000 Spectral Methods in MATLAB. Society for Industrial and Applied Mathematics (SIAM).

Tryggvason, G. \& Aref, H. 1983 Numerical experiments on Hele-Shaw flow with a sharp interface. J. Fluid Mech. 136, 1-30.

Wurlitzer, S., StefFen, P. \& Fischer, T. M. $2000 a$ Line tension in langmuir monolayers probed by point forces. J. Chem. Phys. 112, 5915-5918.

Wurlitzer, S., Steffen, P., Wurlitzer, M., Khattari, Z. \& Fischer, T. M. $2000 b$ Line tension in langmuir monolayers probed by point forces. J. Chem. Phys. 113, 3822-3828.

Zou, L., Basnet, P., Wang, J., Kooljman, E. E. \& Mann, E. K. 2006 Line tension in smectic liquid crystal Langmuir multilayers. Preprint. 